\title{
Reference Gene Expression in Adipose-Derived Stromal Cells Undergoing Adipogenic Differentiation
}

\author{
Carla Dessels, MSc, and Michael Sean Pepper, MBChB, PhD, MD
}

Adipose-derived stromal cells (ASCs) are becoming increasingly attractive as cellular therapy products. Their differentiation potential, the secretion of growth and differentiation factors, and the ability to cryopreserve the cells over extended periods are important features. Changes in experimental conditions result in changes in gene expression, and reverse-transcription quantitative polymerase chain reaction (RT-qPCR) has become an important tool for measuring these changes. There is, however, the potential to introduce technical bias in the process, which can be diminished through the selection of stable reference genes (RGs). Using geNorm software, in this in vitro study we explore the effects that adipogenic differentiation for a 21 day induction period, cryopreservation (freshly isolated ASCs or previously cryopreserved/frozen ASCs), and culture medium supplementation (fetal bovine serum vs. pooled human platelet lysate) have on the stability of 11 RGs. We found that RG stability is markedly affected by the different experimental conditions. Of the RGs assessed, YWHAZ, HPRT, TBP, and ACTB were stably expressed genes under all experimental conditions. We recommend that a panel of stable RGs should be selected before studying gene expression during adipogenesis, and that this is based on the experimental condition(s) being investigated.

Keywords: adipose-derived stromal cells, fetal bovine serum, human platelet lysate, adipogenesis, reference genes, cryopreservation

\section{Impact Statement}

As the use of adipose-derived stromal cells (ASCs) in clinical trials increases, so does the amount of experimental data from research groups, many of which use human ASCs to study adipogenesis in obesity. Different conditions are constantly being applied to ASCs in vitro, to obtain a therapeutic product for potential downstream applications. Few articles have looked at the effect of different conditions on ASC reference gene (RG) expression and stability, which was the aim of this research, as such this article will assist other researchers to make an informed decision about RG selection for gene expression studies using ASCs including those for adipogenesis.

\section{Background}

A DIPOSE-DERIVED STROMAL CELLS (ASCs) are being assessed for their therapeutic potential in clinical trials. ${ }^{1-3}$ To be successful in ASCs' downstream applications, production needs to adhere to good manufacturing processes (GMPs). ${ }^{4}$ The latter can be achieved by replacing animal and chemical products with xeno-free and clinical-grade alternatives. ${ }^{5}$ Altering experimental conditions could alter the ASC product and this will be reflected by changes in gene expression. ${ }^{6-9}$ Attractive features of ASCs include the fact that they can be cryopreserved and stored for prolonged periods of time with minimal loss of their characteristics ${ }^{10-12}$; they remain undifferentiated during expansion ${ }^{13}$; and they have the potential to form adipocytes, chondrocytes, and osteocytes. $^{14,15}$ Reverse-transcription quantitative polymerase chain reaction (RT-qPCR) is the preferred method for measuring gene expression during adipogenesis, ${ }^{15-17}$ and as such requires the selection and validation of a panel of internal controls or reference genes (RGs) for normalization. In this study, we examined the stability of 11 RGs used in adipogenesis studies under different experimental conditions, with the aim of defining which RGs might be the most appropriate.

Institute for Cellular and Molecular Medicine, Department of Immunology, and SAMRC Extramural Unit for Stem Cell Research and Therapy, Faculty of Health Sciences, University of Pretoria, Pretoria, South Africa.

(C) Carla Dessels and Michael Sean Pepper 2019; Published by Mary Ann Liebert, Inc. This Open Access article is distributed under the terms of the Creative Commons License (http://creativecommons.org/licenses/by/4.0), which permits unrestricted use, distribution, and reproduction in any medium, provided the original work is properly cited. 


\section{Methods}

The experimental design and layout can be found in the Supplementary Figure S1.

\section{ASC isolation, cryopreservation, and expansion}

Lipoaspirate samples were collected from four female volunteers undergoing elective liposuction. Stromal vascular fraction (SVF) containing ASCs was isolated from lipoaspirates using established protocols. ${ }^{18,19} \mathrm{SVF}$ was plated at $5 \times 10^{5}$ cells $/ \mathrm{cm}^{2}$ in $80 \mathrm{~cm}^{2}$ (T80) flasks (NUNC ${ }^{\mathrm{TM}}$; Roskilde Site, Kamstrupvej, Denmark). ASCs were maintained in $\alpha$-MEM containing $2 \%(\mathrm{v} / \mathrm{v})$ penicillin $[10,000 \mathrm{U} / \mathrm{mL}]-$ streptomycin $[10,000,8 \mu \mathrm{g} / \mathrm{mL}]\left(\mathrm{p} / \mathrm{s} ;\right.$ GIBCO, Life Technologies ${ }^{\mathrm{TM}}$, New York, NY) and either $10 \%(\mathrm{v} / \mathrm{v})$ fetal bovine serum (FBS; GIBCO, Life Technologies) or pooled human platelet lystate (pHPL) supplemented with preservative-free heparin ([2 U/ $\mathrm{mL}]$; Biochrom, Merck Millipore, Berlin, Germany). The pHPL was manufactured as previously described. ${ }^{20,21}$ At 80 90\% confluence, ASCs were dissociated using tryPLE (Life Technologies) and counted. ASCs at P0 were expanded by plating 5000 cells $/ \mathrm{cm}^{2}$ into T80 flasks and were maintained in $\alpha$-MEM containing $2 \%(\mathrm{v} / \mathrm{v}) \mathrm{p} / \mathrm{s}$ and either $10 \%(\mathrm{v} / \mathrm{v}) \mathrm{pHPL}$ or $10 \%(\mathrm{v} / \mathrm{v}) \mathrm{FBS}$ at $37^{\circ} \mathrm{C}$ in $5 \% \mathrm{CO}_{2}$. Cells remaining after seeding were cryopreserved in $\alpha$-MEM containing $2 \%(\mathrm{v} / \mathrm{v}) \mathrm{p} / \mathrm{s}$ and either $10 \%(\mathrm{v} / \mathrm{v}) \mathrm{pHPL}$ or $10 \%(\mathrm{v} / \mathrm{v})$ FBS and $10 \%(\mathrm{v} / \mathrm{v})$ dimethyl sulfoxide (DMSO) in Cryo. $\mathrm{s}^{\mathrm{TM}}$ tubes (Greiner BioOne $\mathrm{GmbH}$, Frickenhausen, Germany). The cryotubes were placed in a NALGENE ${ }^{\circledR}$ Mr Frosty ${ }^{\mathrm{TM}}$ Cryo $1^{\circ} \mathrm{C}$ freezing container, allowing the cells to cool at a rate of $1^{\circ} \mathrm{C}$ per minute, and subsequently placed at $-80^{\circ} \mathrm{C}$ overnight. ASCs cryopreserved at $\mathrm{P} 0$ were thawed by addition of $\alpha$-MEM containing either $10 \%(\mathrm{v} / \mathrm{v}) \mathrm{pHPL}$ or $10 \%(\mathrm{v} / \mathrm{v})$ FBS to the cryopreservation tubes. The liquid portion containing the cellular fraction was then transferred to a conical tube (Corning, NY); this step was repeated until the ASCs were completely thawed. ASCs were then centrifuged and seeded into T80 flasks and maintained at $37^{\circ} \mathrm{C}$ in $5 \% \mathrm{CO}_{2}$. Before adipogenic differentiation, the immunophenotypic surface markers (Supplementary Data) were assessed using methods previously described. ${ }^{21}$

\section{Adipogenic differentiation}

At P4, ASCs were dissociated and plated for the differentiation experiment as previously described. ${ }^{17}$ At $80 \%$ confluence, freshly isolated ASCs expanded in FBS, previously frozen ASCs expanded in FBS, and previously frozen ASCs expanded in pHPL were induced to differentiate by replacing $\alpha$-MEM supplemented medium with adipogenic induction medium consisting of DMEM (DMEM $1 \times+$ GlutaMAX $^{\mathrm{TM}}$; GIBCO, Thermo Fisher/Life Technologies ${ }^{\mathrm{TM}}$, Grand Island, NY), 2\% p/s, $1 \mu \mathrm{M}$ dexamethasone (SigmaAldrich Chemie, Steinheim, Germany), 0.5 mM 3-iosbutylmethylxanthine (Sigma-Aldrich Chemie), $200 \mu \mathrm{M}$ indomethacin (Sigma-Aldrich Chemie), and $10 \mu \mathrm{g} / \mathrm{mL}$ insulin (human recombinant zinc; GIBCO, Thermo Fisher/Life Technologies), and supplemented with either 10\% (v/v) FBS or 5\% pHPL. Both noninduced (controls) and induced ASCs were dissociated using tryPLE and their viability (Supplementary Table S1) was assessed before RNA isolation on the day of induction (day 0), and on days 1, 7, 14, and 21.

\section{RNA isolation, integrity and quality, and $c D N A$ synthesis}

RNA was isolated from ASCs using RNeasy Minikits (Qiagen, Hilden, Germany) according to the manufacturer's instructions, and quantified on a NanoDrop ${ }^{\circledR}$ ND 1000 spectrophotometer (Thermo Fisher Scientific, Waltham, MA). RNA purity was assessed at an absorbance OD ratio of 260/ 280 and 260/230. Before cDNA synthesis, RNA integrity and quality were assessed using a TapeStation ${ }^{\circledR} 2200$ together with RNA ScreenTape ${ }^{\circledR}$ and Sample Buffer kit according to the manufacturer's instructions (Agilent Technologies; Santa Clara, CA). RNA that had absorbance OD ratios $>2$ and RIN values $>8$ was used for downstream applications. cDNA was synthesized from $100 \mathrm{ng}$ RNA using the SensiFast ${ }^{\mathrm{TM}}$ cDNA synthesis kit (Bioline, London, England) according to the manufacturer's instructions. "No RT controls" were tested and all samples displayed either no amplification or a cycle threshold $(\mathrm{Cq})$ value $>40$.

\section{RGs, primer design and specificity, and amplification efficiency}

Eleven RGs (Table 1) were selected based on data previously published. ${ }^{8,22-24}$ Primers were designed and assessed on the Integrated DNA Technologies (IDT) website. Primers were synthesized by IDT (Coralville, IA), and amplicon specificity was confirmed by the presence of single bands on agarose gel electrophoresis (Supplementary Fig. S2) and single peaks in melt curves. For each of the primers, a six-point standard curve based on a 1:2 dilution series was used and the amplification efficiency (E) and correlation coefficient $\left(R^{2}\right)$ were calculated (Table 1) using BioMark Real-Time PCR Analysis Software 3.1.2 (Fluidigm, South San Francisco, CA).

\section{Reverse-transcription quantitative polymerase chain reaction}

High-throughput RT-qPCR was performed using Biomark ${ }^{\circledR}$ 96:96 dynamic array integrated fluidic circuits (Fluidigm, South San Francisco, CA) according to manufacturer's instructions. In brief, cDNA samples were preamplified using a pool of RG primers and the following cycling conditions: $95^{\circ} \mathrm{C}$ for $2 \mathrm{~min}, 10$ cycles of $95^{\circ} \mathrm{C}$ for $15 \mathrm{~s}$, and $60^{\circ} \mathrm{C}$ for $4 \mathrm{~min}$. Preamplified products were cleaned by Exonuclease I (Inqaba Biotec, Pretoria, South Africa) dilution. The following conditions were used: $95^{\circ} \mathrm{C}$ for $10 \mathrm{~min}, 35$ cycles of $95^{\circ} \mathrm{C}$ for $15 \mathrm{~s}$, and $60^{\circ} \mathrm{C}$ for $30 \mathrm{~s}$. All samples were run as six technical replicates. "No template controls" and "no primer controls" were included to determine genomic amplification or the presence of primer dimers. Standard curves were run on each circuit.

\section{RG stability}

Samples were divided into three experimental groups: (1) freshly isolated ASCs expanded in FBS (fresh FBS), (2) previously cryopreserved ASCs expanded in FBS (frozen FBS), and (3) previously cryopreserved ASCs expanded in pHPL (frozen HPL). Each group consisted of four biological replicates with nine samples each. The nine samples comprised noninduced (control) samples collected on days $0,1,7,14$, and 21 ; induced samples were collected on days 1, 7, 14, and 21. Comparisons were performed between different subsets of 


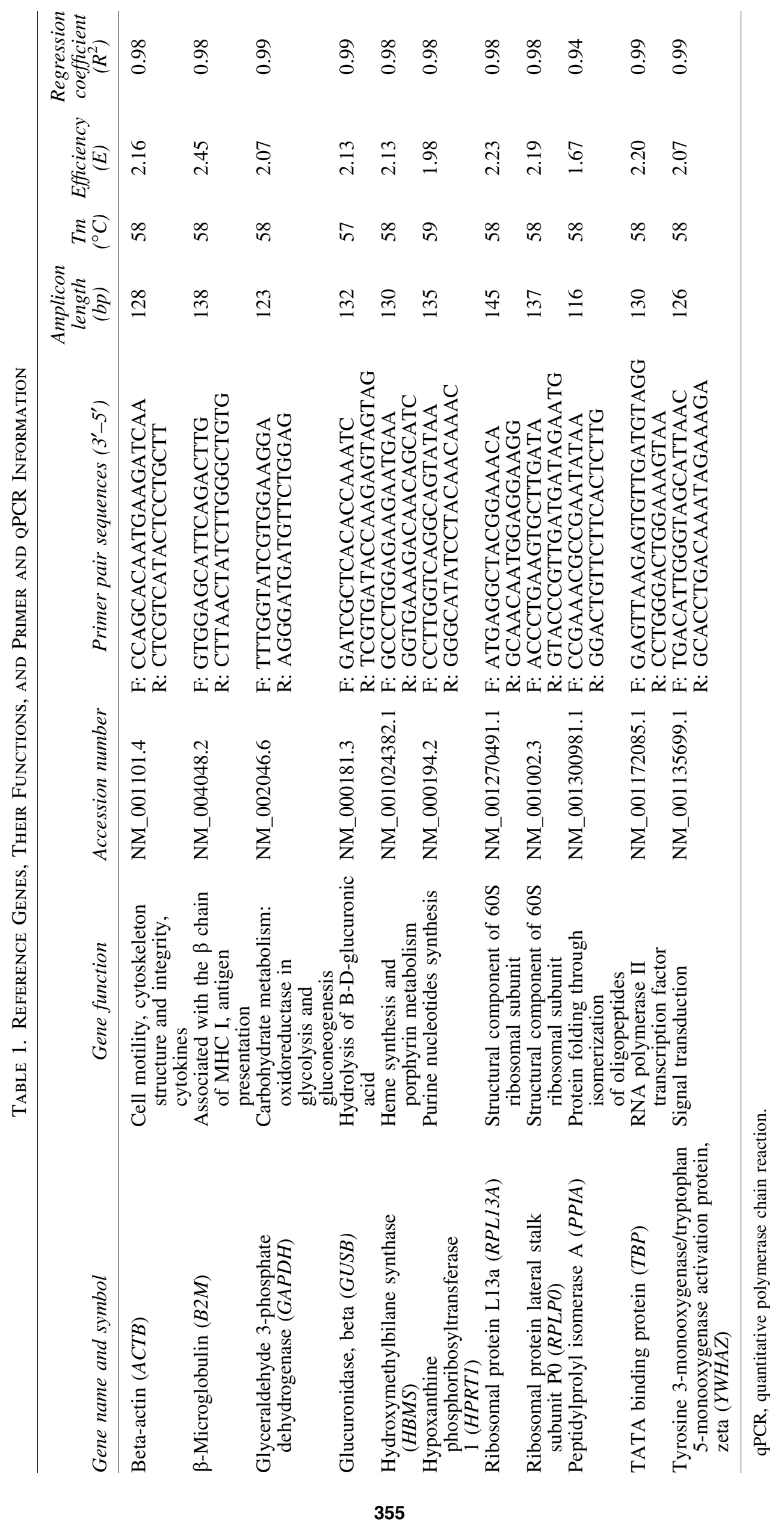


Table 2. Assumptions Made During the Adipogenic Differentiation Assay and Comparisons Used to Test the Stability of the Reference Genes Under the Different Assumptions

\begin{tabular}{|c|c|c|}
\hline Assumptions & Comparison & Example \\
\hline $\begin{array}{l}\text { Does time in culture affect RG stability } \\
\text { Growth kinetic affects } \\
\text { Comparison over time points in the same } \\
\text { condition }\end{array}$ & $\begin{array}{l}\text { Comparison of day } 0 \text { (D0) through } \\
\text { to day } 21 \text { (D21) in either the control } \\
\text { or the induced ASCs for the same } \\
\text { condition }\end{array}$ & $\begin{array}{l}\text { Day } 1 \text { (D1) control frozen pHPL } \\
\text { ASCs vs. day } 7 \text { (D7) control } \\
\text { frozen pHPL ASCs } \\
\text { Day } 14 \text { (D14) induced fresh } \\
\text { FBS ASCs vs. day } 21 \text { (D21) } \\
\text { induced fresh FBS ASCs }\end{array}$ \\
\hline $\begin{array}{l}\text { Does adipogenesis affect RG stability } \\
\text { Induction affects } \\
\text { Comparison between induced and controls } \\
\text { in the same condition }\end{array}$ & $\begin{array}{l}\text { Comparison of the induced and control } \\
\text { samples on the same day for each } \\
\text { condition }\end{array}$ & $\begin{array}{l}\text { Induced day } 1 \text { (D1) frozen FBS } \\
\text { ASCs vs. control day } 1 \text { (D1) } \\
\text { frozen FBS ASCs } \\
\text { Induced day } 21 \text { (D21) frozen } \\
\text { pHPL ASCs vs. control day } \\
21 \text { (D21) frozen pHPL ASCs }\end{array}$ \\
\hline $\begin{array}{l}\text { Does cryopreservation affect RG stability } \\
\text { Cryopreservation affects } \\
\text { Comparison between fresh and frozen } \\
\text { ASCs in the same medium }\end{array}$ & $\begin{array}{l}\text { Comparison of the induced samples } \\
\text { of fresh FBS ASCs and frozen FBS } \\
\text { ASCs on the same day }\end{array}$ & $\begin{array}{l}\text { Induced day } 7 \text { (D7) frozen FBS } \\
\text { ASCs vs. induced day } 7 \text { (D7) } \\
\text { fresh FBS ASCs }\end{array}$ \\
\hline $\begin{array}{l}\text { Does the medium affect RG stability by } \\
\text { comparing: } \\
\text { Media supplementation affects } \\
\text { Comparison between FBS ASCs and pHPL } \\
\text { ASCs in the same cryopreservation state }\end{array}$ & $\begin{array}{l}\text { Comparison between the induced } \\
\text { samples of frozen FBS and frozen } \\
\text { pHPL ASCs on the same day }\end{array}$ & $\begin{array}{l}\text { Induced day } 7 \text { (D7) frozen FBS } \\
\text { ASCs vs. induced day } 7 \text { (D7) } \\
\text { frozen pHPL ASCs }\end{array}$ \\
\hline
\end{tabular}

ASC, adipose-derived stromal cell; FBS, fetal bovine serum; p/HPL, pooled human platelet lysate; RG, reference gene.

samples, between days, between induced and control samples, between cryopreserved and noncryopreserved ASCs, and between medium supplemented with either pHPL or FBS based on the assumptions we make in the adipogenic differentiation assay (Table 2). RG stability was determined using geNorm ${ }^{25}$ and comparisons were performed using the R-based NormqPCR package. ${ }^{26}$ The input data for geNorm required relative expression values. In this study, relative expression was calculated by converting the raw $\mathrm{Cq}$ values into relative expression values using the formula $E^{-\Delta \mathrm{Cq}}$, where $\Delta \mathrm{Cq}$ is specific $\mathrm{RG} \mathrm{Cq}$ value-minimum corresponding $\mathrm{RG} \mathrm{Cq}$ and either $100 \%$ efficiency was assumed $(E=2)$ or RG specific efficiencies (SEs) were used.

\section{Statistical analysis}

All data and statistical analyses were performed in RStudio ( $\mathrm{R}$ Version 3.3.2). ${ }^{27}$ Descriptive statistics were calculated from the raw $\mathrm{Cq}$ values for all experimental conditions. To compare the means between the different control and induced groups in the same medium type for a specific RG or between medium types for a specific RG under a specific condition or at a specific time point, a Mann-Whitney U test was employed. To compare the means between days for each condition and medium type, a Kruskal-Wallis test, followed by a Dunn's post hoc multiple comparisons test with a Bonferroni correction, was employed. The significance level for all statistical analyses was set at $\alpha=0.05$, and a value of $p<0.05$ was considered to be significant.

\section{Results}

\section{$R G$ expression}

Levels of expression of the 11 RGs were determined using $\mathrm{Cq}$ values. The lowest average $\mathrm{Cq}$ value was for $B 2 M$
$(6.7 \pm 1.19)$, and the highest value was for $H B M S$ $(15.1 \pm 1.08)$. The least variable $\mathrm{Cq}$ values were seen for $R P L P O(0.82)$ and the most variable $\mathrm{Cq}$ values were seen for PPIA (2.19). ACTB, B2M, GAPDH, RPL13A, RPLPO, and $Y W H A Z$ had the highest expression levels, whereas $G U S B$, HBMS, HPRT, PPIA, and TBP had the lowest (Fig. 1).

\section{Effect of adipogenic differentiation on RG expression and stability}

Adipogenesis was measured using flow cytometry and fluorescence microscopy by Nile red staining as previously described. ${ }^{17}$ Adipogenesis was induced in all experimental groups as shown by the increase in Nile red positivity percentage (Supplementary Fig. S3) and by the appearance of lipid droplets by day 21 of adipogenic induction (Supplementary Fig. S4). We first examined the variability and stability of the 11 RGs in the control and induced samples for each time point (day $0,1,7,14$, and 21) for the three groups: fresh FBS, frozen FBS, and frozen HPL. Variability and significant differences were found between the $\mathrm{Cq}$ values in all three groups when comparing the effect and the kinetics of differentiation (Supplementary Figs. S5-S7 and Supplementary Table S2).

RG stability was measured at each time point for both control and induced samples for each of the groups (Table 3$)$, assuming either $100 \%$ efficiency $(E=2)$ or using the SEs for each of the RGs. For the fresh FBS group, when $E=2, B 2 M, G A P D H$, and $Y W H A Z$ appeared more regularly in the higher rankings, whereas PPIA and RPLPO appeared more regularly in the lower rankings. When SE was used, $G U S B, T B P$, and $Y W H A Z$ appeared more regularly in the higher rankings, whereas $P P I A$ and $R P L P O$ appeared more regularly in the lower rankings. For the frozen FBS group, when $E=2, G A P D H, H B M S, H P R T$, and $Y W H A Z$ appeared 


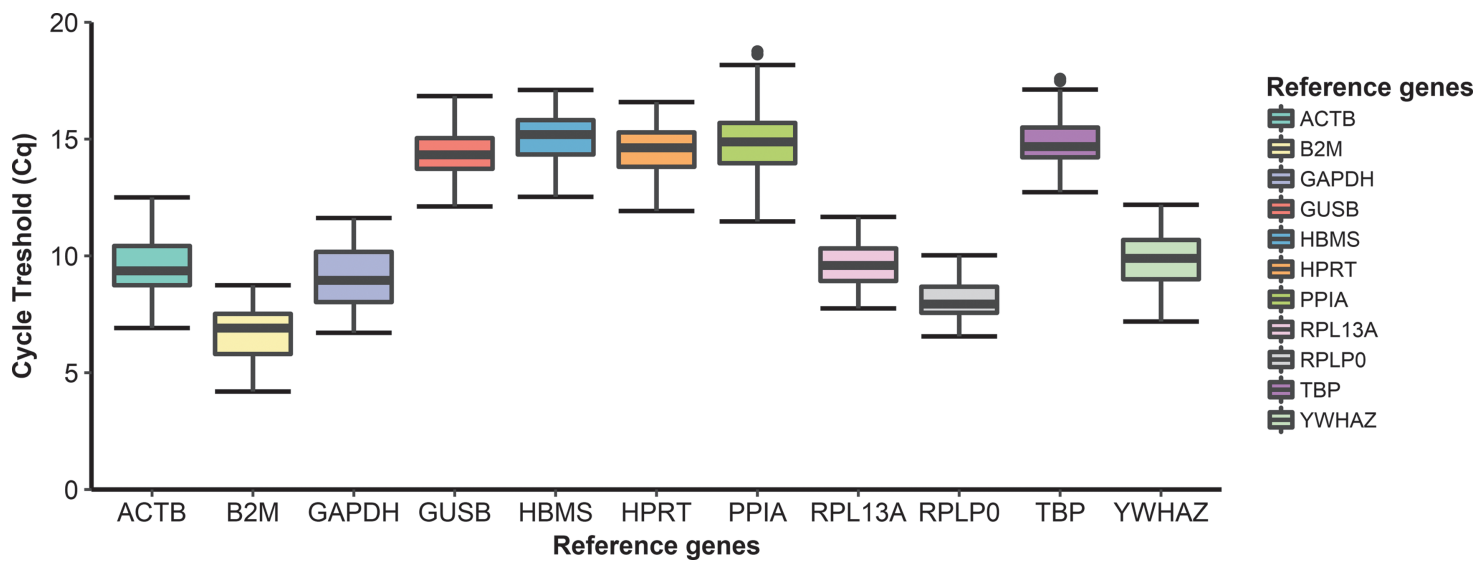

FIG. 1. Box and whisker plots of the Cq values for the $11 \mathrm{RGs}$ assessed. Boxes extend from the first to third quartiles with the median shown as a solid black line intersecting the box; the whiskers extend to the minimum and maximum values that lie within $1.5 \times$ the IQR. Data points beyond the whiskers represent outliers. Sample size is $n=269$ and represents all biological replicates across all experimental conditions. RG, reference gene; IQR, interquartile range; Cq, cycle threshold.

more regularly in the higher rankings, whereas $G U S B$ and $R P L P O$ appeared more regularly in the lower rankings. When SE was used, B2M, HBMS, HPRT, and YWHAZ appeared more regularly in the higher ranking, whereas $P P I A$ and $R P L P O$ appeared more regularly in the lower rankings. For the frozen HPL group, when $E=2, H P R T$ and $Y W H A Z$ appeared more regularly in the higher rankings, whereas $A C T B, P P I A$, and RPLPO appeared more regularly in the lower rankings. When the SE was used, HBMS, HPRT, and $Y W H A Z$ appeared more regularly in the higher rankings, whereas $A C T B, P P I A$, and RPLPO appeared more regularly in the lower rankings. To determine the overall stability for a specific group, kinetics and differentiation (controls and induced) samples were grouped together and ranked. For the fresh FBS group, the efficiency method's stability rankings were identical, where TBP, YWHAZ, HPRT, and $A C T B$ had the greatest stability and GUSB, B2M, RPL13A, and PPIA were the least stable. In the frozen FBS group, $H P R T$, $Y W H A Z, H B M S$, and $A C T B$ were the most stable for both efficiency methods, whereas when $E=2, R P L 13 A, G U S B$, $B 2 M$, and $R P L P O$ were least stable, and when SE was used, $G U S B, R P L 13 A, P P I A$, and RPLPO were the least stable. In the frozen HPL group, B2M, GUSB, TBP, and YWHAZ displayed the highest stability and were identical for both efficiency methods, whereas GAPDH, ACTB, RPL13A, and $R P L P O$ were the least stable when $E=2$, and $A C T B, P P I A$, $R P L 13 A$, and RPL13A were the least stable for SE.

\section{Effect of cryopreservation on RG expression and stability}

We next examined the effect of cryopreservation on $\mathrm{RG}$ expression and stability by grouping all the samples together for the fresh FBS and frozen FBS groups. When compared, the frozen FBS group had significantly higher $\mathrm{Cq}$ values for all RGs, except for PPIA and RPLPO (Fig. 2).

RG stability was measured for each time point in both control and induced samples for each of the groups (Table 4; Fresh FBS vs. Frozen FBS) using both efficiency methods. When $E=2, H P R T, H B M S$, and YWHAZ appeared more regularly in the higher rankings, whereas $G U S B, B 2 M$, and
$R P L P O$ appeared more regularly in the lower rankings. When the SE was used, HBMS, HPRT, and YWHAZ appeared more regularly in the higher rankings, whereas RPLPO and PPIA appeared more regularly in the lower rankings. When all samples were combined and $E=2$, $H P R T, Y W H A Z, A C T B$, and TBP were the most stable and $B 2 M$, PPIA, RPLPO, and RPL13A were the least stable. When the SE was used, HPRT, YWHAZ, TBP, and $A C T B$ were the most stable, whereas $R P L P 0, B 2 M, R P L 13 A$, and PPIA were the least stable.

\section{Effect of medium on RG expression and stability}

We examined the effect that expansion medium had on RG expression and stability by grouping all the samples for the frozen FBS and frozen HPL groups. The frozen FBS group had significantly higher $\mathrm{Cq}$ values for $A C T B, G A P D H$, and $H P R T$, whereas the frozen HPL group had significantly higher $\mathrm{Cq}$ values for $B 2 M$ and GUSB (Fig. 3).

RG stability was measured for each time point for both control and induced samples for each of the groups (Table 4; Frozen FBS vs. Frozen HPL) using both efficiency methods. When $E=2, A C T B, H P R T, H B M S$, and YWHAZ appeared more regularly in the higher rankings, whereas GUSB, RPLPO, RPLP13A and PPIA appeared more regularly in the lower rankings. When the SE was used, $H B M S, H P R T$, and $Y W H A Z$ appeared more regularly in the higher rankings, whereas RPLPO, RPL13A, and PPIA appeared more regularly in the lower ranking. When all the samples were combined and $E=2, H B M S, H P R T, P P I A$, and $G A P D H$ were the most stable and RPL13A, GUSB, B2M, and RPLPO were the least stable. When the SE was used, HPRT, $Y W H A Z, H B M S$, and $T B P$ were the most stable, whereas $B 2 M, G U S B, R P L 13 A$, and RPLPO were the least stable.

\section{Discussion}

ASCs can be cryopreserved for future use with apparently limited alterations to their inherent characteristics. ${ }^{1,10}$ ASCs are being assessed a number of settings in the hope that a cell therapy product will be identified. Independent of their therapeutic efficacy, ASC products need to adhere to GMP 
Table 3. Specific Efficiency and 100\% Efficiency Reference Gene Stability Ranking and Values For the 11 Reference Genes in the Fresh FBS, Frozen FBS, and Frozen HPL ExPanded ASCs at Each Time Point in the Control and Induced Samples

\begin{tabular}{|c|c|c|c|c|c|c|c|c|c|c|c|c|c|c|}
\hline \multirow[b]{3}{*}{ Day } & \multirow[b]{3}{*}{ Differentiation } & \multirow[b]{3}{*}{ Rank } & \multicolumn{4}{|c|}{ Fresh FBS } & \multicolumn{4}{|c|}{ Frozen FBS } & \multicolumn{4}{|c|}{$p H P L$} \\
\hline & & & \multicolumn{2}{|c|}{$\begin{array}{c}100 \% \\
\text { efficiency }\end{array}$} & \multicolumn{2}{|c|}{$\begin{array}{l}\text { Specific } \\
\text { efficiency }\end{array}$} & \multicolumn{2}{|c|}{$\begin{array}{c}100 \% \\
\text { efficiency }\end{array}$} & \multicolumn{2}{|c|}{$\begin{array}{l}\text { Specific } \\
\text { efficiency }\end{array}$} & \multicolumn{2}{|c|}{$\begin{array}{c}100 \% \\
\text { efficiency }\end{array}$} & \multicolumn{2}{|c|}{$\begin{array}{l}\text { Specific } \\
\text { efficiency }\end{array}$} \\
\hline & & & $R G$ & $M$ & $R G$ & $M$ & $R G$ & $M$ & $R G$ & $M$ & $R G$ & $M$ & $R G$ & $M$ \\
\hline \multirow[t]{11}{*}{0} & Control & 1 & $B 2 M$ & 0.01 & $G U S B$ & 0.01 & $A C T B$ & 0.02 & $B 2 M$ & 0.02 & $H P R T$ & 0.03 & $G A P D H$ & 0.02 \\
\hline & & 2 & RPL13A & 0.01 & $T B P$ & 0.01 & PPIA & 0.02 & HPRT & 0.02 & $R P L P O$ & 0.03 & $R P L P O$ & 0.02 \\
\hline & & 3 & $G A P D H$ & 0.02 & $B 2 M$ & 0.02 & $H P R T$ & 0.03 & $A C T B$ & 0.03 & $Y W H A Z$ & 0.04 & $Y W H A Z$ & 0.04 \\
\hline & & 4 & $H P R T$ & 0.03 & $H P R T$ & 0.03 & $H B M S$ & 0.04 & $H B M S$ & 0.03 & $G A P D H$ & 0.04 & $H B M S$ & 0.04 \\
\hline & & 5 & $Y W H A Z$ & 0.03 & RPL13A & 0.03 & $Y W H A Z$ & 0.05 & $Y W H A Z$ & 0.04 & $H B M S$ & 0.04 & $T B P$ & 0.06 \\
\hline & & 6 & $T B P$ & 0.04 & $G A P D H$ & 0.04 & $G A P D H$ & 0.05 & $G A P D H$ & 0.04 & $T B P$ & 0.05 & RPL13A & 0.06 \\
\hline & & 7 & $A C T B$ & 0.04 & $Y W H A Z$ & 0.04 & $B 2 M$ & 0.05 & $R P L 13 A$ & 0.05 & RPL13A & 0.06 & $H P R T$ & 0.06 \\
\hline & & 8 & $G U S B$ & 0.05 & $A C T B$ & 0.05 & $R P L 13 A$ & 0.06 & $T B P$ & 0.06 & $G U S B$ & 0.07 & $G U S B$ & 0.07 \\
\hline & & 9 & $H B M S$ & 0.05 & $R P L P O$ & 0.05 & $T B P$ & 0.07 & $G U S B$ & 0.06 & $B 2 M$ & 0.07 & $B 2 M$ & 0.08 \\
\hline & & 10 & PPIA & 0.06 & $H B M S$ & 0.06 & $G U S B$ & 0.08 & $R P L P O$ & 0.06 & PPIA & 0.08 & $A C T B$ & 0.09 \\
\hline & & 11 & $R P L P O$ & 0.07 & PPIA & 0.08 & $R P L P O$ & 0.08 & PPIA & 0.07 & $A C T B$ & 0.09 & PPIA & 0.1 \\
\hline 1 & Con & 1 & $G A P D H$ & 0.04 & $G A P D H$ & 0.04 & $G A P D H$ & 0.02 & $G A P D H$ & 0.01 & $B 2 M$ & 0.02 & $G A P D H$ & 0.01 \\
\hline & & 2 & $Y W H A Z$ & 0.04 & $Y W H A Z$ & 0.04 & $Y W H A Z$ & 0.02 & $Y W H A Z$ & 0.01 & $H B M S$ & 0.02 & $Y W H A Z$ & 0.01 \\
\hline & & 3 & $T B P$ & 0.06 & $T B P$ & 0.07 & $H P R T$ & 0.02 & $B 2 M$ & 0.02 & $R P L P O$ & 0.02 & $B 2 M$ & 0.01 \\
\hline & & 4 & RPL13A & 0.07 & RPL13A & 0.08 & $B 2 M$ & 0.02 & $H P R T$ & 0.02 & $G U S B$ & 0.02 & $H B M S$ & 0.01 \\
\hline & & 5 & $B 2 M$ & 0.08 & $B 2 M$ & 0.08 & $R P L 13 A$ & 0.03 & RPL13A & 0.02 & $R P L 13 A$ & 0.02 & $G U S B$ & 0.02 \\
\hline & & 6 & $H B M S$ & 0.09 & $H B M S$ & 0 & $T B P$ & 0.03 & $H B M S$ & 3 & $G A P D H$ & 0.03 & RPL13A & 0.02 \\
\hline & & 7 & $G U S B$ & 0.1 & GUSB & 0 & $H B M S$ & 0.04 & $A C T B$ & 0.03 & $T B P$ & 0.03 & $R P L P O$ & 0.02 \\
\hline & & 8 & $A C T B$ & 0.1 & $A C T B$ & 0.11 & $A C T B$ & 0.04 & $T B P$ & 0.03 & $Y W H A Z$ & 0.03 & $T B P$ & 0.02 \\
\hline & & 9 & $H P R T$ & 0.12 & HPRT & 0.12 & PPIA & 0.05 & $G U S B$ & 0.04 & $H P R T$ & 0.03 & $H P R T$ & 0.03 \\
\hline & & 10 & RPLPO & 0.13 & $R P L P O$ & 0.13 & RPLPO & 0.06 & $R P L P O$ & 0.05 & PPIA & 0.03 & $A C T B$ & 0.04 \\
\hline & & 11 & PPIA & 0.17 & PPIA & 0.17 & $G U S B$ & 0.06 & PPIA & 0.06 & $A C T B$ & 0.04 & PPIA & 0.05 \\
\hline 1 & Indu & 1 & $G A P D H$ & 0.02 & $T B P$ & 0.01 & $G U S B$ & 0.02 & $H P R T$ & 0.01 & $H P R T$ & 0.02 & $H P R T$ & 0.02 \\
\hline & & 2 & RPL13A & 0.02 & $Y W H A Z$ & 0.01 & $T B P$ & 0.02 & $Y W H A Z$ & 0.01 & $Y W H A Z$ & 0.02 & $Y W H A Z$ & 0.02 \\
\hline & & 3 & $R P L P O$ & 0.03 & $H P R T$ & 0.01 & $R P L 13 A$ & 0.03 & $A C T B$ & 0.01 & RPL13A & 0.02 & $R P L 13 A$ & 0.02 \\
\hline & & 4 & $G U S B$ & 0.04 & $B 2 M$ & 0.05 & $G A P D H$ & 0.04 & $B 2 M$ & 0.02 & $G U S B$ & 0.02 & $G U S B$ & 0.02 \\
\hline & & 5 & $B 2 M$ & 0.05 & $G U S B$ & 0.07 & $B 2 M$ & 0.04 & $R P L 13 A$ & 0.04 & $B 2 M$ & 0.03 & $T B P$ & 0.03 \\
\hline & & 6 & $H P R T$ & 0.07 & $R P L P O$ & & $A C T B$ & 0.05 & $T B P$ & 0.05 & $H B M S$ & 0.04 & $H B M S$ & 0.03 \\
\hline & & 7 & $Y W H A Z$ & 0.08 & $G A P D H$ & & $Y W H A Z$ & 0.06 & $G U S B$ & & $T B P$ & 4 & $B 2 M$ & 0.03 \\
\hline & & 8 & $T B P$ & 0.09 & RPL13A & 0.09 & $H P R T$ & 0.06 & $G A P D H$ & 0.05 & $G A P D H$ & 0.05 & $A C T B$ & 0.04 \\
\hline & & 9 & $H B M S$ & 0.1 & $H B M S$ & 0.1 & $H B M S$ & 0.06 & $H B M S$ & 0.06 & $A C T B$ & 0.05 & $G A P D H$ & 0.04 \\
\hline & & 10 & $A C T B$ & 0.11 & $A C T B$ & 0.11 & PPIA & 0.07 & PPIA & 0.07 & PPIA & 0.06 & $R P L P O$ & 0.05 \\
\hline & & 11 & PPIA & 0.12 & PPIA & 0.12 & $R P L P O$ & 0.09 & $R P L P O$ & 0.09 & $R P L P O$ & 0.07 & PPIA & 0.07 \\
\hline 7 & Control & 1 & $B 2 M$ & 0.04 & $G U S B$ & 0.02 & $H B M S$ & 0.02 & RPL13A & 0.02 & $B 2 M$ & 0.01 & $B 2 M$ & 0 \\
\hline & & 2 & $T B P$ & 0.04 & $H B M S$ & 0.02 & $Y W H A Z$ & 0.02 & $T B P$ & 0.02 & $H B M S$ & 0.01 & $H B M S$ & 0 \\
\hline & & 3 & $Y W H A Z$ & 0.05 & $T B P$ & 0.06 & $A C T B$ & 0.02 & $G U S B$ & 0.03 & $Y W H A Z$ & 0.01 & $Y W H A Z$ & 0.01 \\
\hline & & 4 & $A C T B$ & 0.06 & $Y W H A Z$ & 0.07 & $H P R T$ & 0.03 & $B 2 M$ & 0.03 & $H P R T$ & 0.02 & $H P R T$ & 0.02 \\
\hline & & 5 & $H P R T$ & 0.07 & $B 2 M$ & 0.08 & PPIA & 0.03 & $G A P D H$ & 0.04 & $G A P D H$ & 0.02 & $G A P D H$ & 0.02 \\
\hline & & 6 & $H B M S$ & 0.08 & $A C T B$ & 0.09 & $G A P D H$ & 0.04 & $Y W H A Z$ & 0.04 & RPL13A & 0.03 & RPL13A & 0.02 \\
\hline & & 7 & $G U S B$ & 0.09 & $H P R T$ & 0.09 & $R P L 13 A$ & 0.05 & $H B M S$ & 0.04 & $A C T B$ & 0.03 & $T B P$ & 0.03 \\
\hline & & 8 & $G A P D H$ & 0.09 & $R P L P O$ & 0 . & $T B P$ & 0.06 & $A C T B$ & 0.05 & $T B P$ & 0.04 & $A C T B$ & 0.03 \\
\hline & & 9 & $R P L P O$ & 0.1 & $G A P D H$ & & $G U S B$ & 0.06 & $H P R T$ & 0.05 & $G U S B$ & 0.04 & PPIA & 0.03 \\
\hline & & 10 & RPL13A & 0.11 & RPL13A & 0.11 & $B 2 M$ & 0.07 & PPIA & 0.05 & PPIA & 0.04 & $G U S B$ & 0.04 \\
\hline & & 11 & PPIA & 0.15 & PPIA & 0.15 & RPLPO & 0.08 & $R P L P O$ & 0.06 & RPLPO & 0.06 & RPLPO & 0.05 \\
\hline 7 & Induced & 1 & $A C T B$ & 0.02 & $G U S B$ & & & 0.01 & $A C T B$ & 0.01 & $H P R T$ & 0.02 & $H P R T$ & 0.01 \\
\hline & & 2 & $Y W H A Z$ & 0.02 & $T B P$ & 0.04 & $H B M S$ & 0.01 & $H P R T$ & 0.01 & $Y W H A Z$ & 0.02 & $Y W H A Z$ & 0.01 \\
\hline & & 3 & $T B P$ & 0.03 & $B 2 M$ & 0.04 & $H P R T$ & 0.01 & $H B M S$ & 0.01 & $B 2 M$ & 0.03 & $B 2 M$ & 0.02 \\
\hline & & 4 & PPIA & 0.04 & $A C T B$ & 0.05 & $Y W H A Z$ & 0.02 & $Y W H A Z$ & 0.02 & $G U S B$ & 0.03 & $T B P$ & 0.03 \\
\hline & & 5 & $G A P D H$ & 0.04 & $Y W H A Z$ & 0.05 & PPIA & 0.02 & $B 2 M$ & 0.02 & $T B P$ & 0.04 & $H B M S$ & 0.04 \\
\hline & & 6 & $G U S B$ & 0.05 & $G A P D H$ & 0.06 & $B 2 M$ & 0.02 & $G U S B$ & 0.03 & $H B M S$ & 0.05 & $G U S B$ & 0.04 \\
\hline & & 7 & $B 2 M$ & 0.06 & $H P R T$ & 0.07 & $T B P$ & 0.03 & $G A P D H$ & 0.03 & $A C T B$ & 0.06 & $A C T B$ & 0.05 \\
\hline & & 8 & $H P R T$ & 0.07 & PPIA & 0.08 & $G A P D H$ & 0.03 & $T B P$ & 0.03 & PPIA & 0.06 & $G A P D H$ & 0.06 \\
\hline & & 9 & $H B M S$ & 0.08 & $R P L P O$ & 0.09 & $G U S B$ & 0.04 & RPL13A & 0.04 & $G A P D H$ & 0.07 & $R P L 13 A$ & 0.08 \\
\hline & & 10 & $R P L P O$ & 0.09 & $H B M S$ & 0.09 & $R P L 13 A$ & 0.04 & PPIA & 0.05 & $R P L 13 A$ & 0.09 & PPIA & 0.1 \\
\hline & & 11 & RPL13A & 0.1 & RPL13A & 0.11 & $R P L P O$ & 0.06 & RPLPO & 0.06 & RPLPO & 0.12 & RPLPO & 0.13 \\
\hline
\end{tabular}


Table 3. (Continued)

\begin{tabular}{|c|c|c|c|c|c|c|c|c|c|c|c|c|c|c|}
\hline \multirow[b]{3}{*}{ Day } & \multirow[b]{3}{*}{ Differentiation } & \multirow[b]{3}{*}{ Rank } & \multicolumn{4}{|c|}{ Fresh FBS } & \multicolumn{4}{|c|}{ Frozen FBS } & \multicolumn{4}{|c|}{$p H P L$} \\
\hline & & & \multicolumn{2}{|c|}{$\begin{array}{c}100 \% \\
\text { efficiency }\end{array}$} & \multicolumn{2}{|c|}{$\begin{array}{l}\text { Specific } \\
\text { efficiency }\end{array}$} & \multicolumn{2}{|c|}{$\begin{array}{c}100 \% \\
\text { efficiency }\end{array}$} & \multicolumn{2}{|c|}{$\begin{array}{l}\text { Specific } \\
\text { efficiency }\end{array}$} & \multicolumn{2}{|c|}{$\begin{array}{c}100 \% \\
\text { efficiency }\end{array}$} & \multicolumn{2}{|c|}{$\begin{array}{l}\text { Specific } \\
\text { efficiency }\end{array}$} \\
\hline & & & $R G$ & $M$ & $R G$ & $M$ & $R G$ & $M$ & $R G$ & $M$ & $R G$ & $M$ & $R G$ & $M$ \\
\hline \multirow[t]{11}{*}{14} & $\mathrm{Co}$ & 1 & $H B M S$ & 0.03 & $H B M S$ & 0.03 & $H B M S$ & 0.01 & $H B M S$ & 0.01 & $G U S B$ & 0.01 & $G U S B$ & 0.01 \\
\hline & & 2 & $Y W H A Z$ & 0.03 & $Y W H A Z$ & 0.03 & $H P R T$ & 0.01 & $H P R T$ & 0.01 & $H P R T$ & 0.01 & $H P R T$ & 0.01 \\
\hline & & 3 & $R P L 13 A$ & 0.03 & RPL13A & 0.03 & $G A P D H$ & 0.01 & $G A P D H$ & 0.01 & $Y W H A Z$ & 0.01 & $Y W H A Z$ & 0.01 \\
\hline & & 4 & $A C T B$ & 0.04 & $A C T B$ & 0.04 & $Y W H A Z$ & 0.02 & $Y W H A Z$ & 0.02 & $H B M S$ & 0.02 & $H B M S$ & 0.01 \\
\hline & & 5 & $H P R T$ & 0.04 & $T B P$ & 0.04 & $A C T B$ & 0.02 & $A C T B$ & 0.02 & $B 2 M$ & 0.02 & $B 2 M$ & 0.02 \\
\hline & & 6 & $G A P D H$ & 0.04 & $H P R T$ & 0.05 & $B 2 M$ & 0.03 & $B 2 M$ & 0.03 & RPL13A & 0.02 & RPL13A & 0.02 \\
\hline & & 7 & $T B P$ & 0.05 & $G A P D H$ & 0.05 & RPL13A & 0.04 & $T B P$ & 0.03 & PPIA & 0.02 & PPIA & 0.02 \\
\hline & & 8 & $R P L P O$ & 0.05 & $R P L P O$ & 0.05 & $T B P$ & 0.04 & $R P L 13 A$ & 0.04 & $G A P D H$ & 0.02 & $G A P D H$ & 0.03 \\
\hline & & 9 & $G U S B$ & 0.06 & $G U S B$ & 0.06 & $G U S B$ & 0.05 & $G U S B$ & 0.04 & $T B P$ & 0.03 & $A C T B$ & 0.03 \\
\hline & & 10 & $B 2 M$ & 0.08 & $B 2 M$ & 0.08 & $R P L P O$ & 0.06 & $R P L P O$ & 0.05 & $A C T B$ & 0.04 & $T B P$ & 0.04 \\
\hline & & 11 & PPIA & 0.09 & PPIA & 0.1 & PPIA & 0.06 & PPIA & 0.07 & RPLPO & 0.04 & $R P L P O$ & 0.04 \\
\hline 14 & Indu & 1 & $G U S B$ & 0.02 & $T B P$ & 0.02 & $G A P D H$ & 0.01 & $G A P D H$ & 0.01 & $A C T B$ & 0.01 & $A C T B$ & 0.01 \\
\hline & & 2 & $Y W H A Z$ & 0.02 & $Y W H A Z$ & 0.02 & $H B M S$ & 0.01 & $H B M S$ & 0.01 & $G A P D H$ & 0.01 & $Y W H A Z$ & 0.01 \\
\hline & & 3 & $H P R T$ & 0.03 & $G U S B$ & 0.02 & $Y W H A Z$ & 0.01 & $Y W H A Z$ & 0.01 & $G U S B$ & 0.01 & $H B M S$ & 0.01 \\
\hline & & 4 & $T B P$ & 0.03 & $G A P D H$ & 0.03 & $B 2 M$ & 0.01 & $B 2 M$ & 0.01 & $Y W H A Z$ & 0.02 & $G A P D H$ & 0.01 \\
\hline & & 5 & $B 2 M$ & 0.03 & $H P R T$ & 0.04 & $H P R T$ & 0.01 & $H P R T$ & 0.01 & $H B M S$ & 0.02 & $G U S B$ & 0.02 \\
\hline & & 6 & $G A P D H$ & 0.04 & $R P L P O$ & 0.04 & $A C T B$ & 0.01 & $A C T B$ & 0.01 & $B 2 M$ & 0.02 & $B 2 M$ & 0.02 \\
\hline & & 7 & $H B M S$ & 0.04 & $B 2 M$ & 0.04 & GUSB & 0.02 & GUSB & 0.02 & $H P R T$ & 0.03 & $H P R T$ & 0.03 \\
\hline & & 8 & RPLPO & 0.05 & $H B M S$ & 0.05 & PPIA & 0.02 & PPIA & 0.02 & $T B P$ & 0.04 & $T B P$ & 0.04 \\
\hline & & 9 & $A C T B$ & 0.06 & $A C T B$ & 0.05 & $T B P$ & 0.03 & $T B P$ & 0.03 & PPIA & 0.05 & PPIA & 0.06 \\
\hline & & 10 & PPIA & 0.08 & PPIA & 0.08 & RPL13A & 0.03 & RPL13A & 0.03 & RPL13A & 0.07 & RPL13A & 0.07 \\
\hline & & 11 & RPL13A & 0.11 & RPL13A & 0.11 & $R P L P O$ & 0.05 & $R P L P O$ & 0.05 & $R P L P O$ & 0.1 & RPLPO & 0.1 \\
\hline 21 & Control & 1 & $G A P D H$ & 0.01 & $A C T B$ & 0.02 & $H B M S$ & 0.01 & $A C T B$ & 0.01 & $H B M S$ & 0.01 & $H B M S$ & 0.01 \\
\hline & & 2 & $H B M S$ & 0.01 & $H P R T$ & 0.02 & $H P R T$ & 0.01 & $H B M S$ & 0.01 & $H P R T$ & 0.01 & $H P R T$ & 0.01 \\
\hline & & 3 & $H P R T$ & 0.02 & $H B M S$ & 0.02 & $G A P D H$ & 0.01 & PPIA & 0.01 & $B 2 M$ & 0.02 & $B 2 M$ & 0.02 \\
\hline & & 4 & $A C T B$ & 0.02 & $G A P D H$ & 0.02 & $Y W H A Z$ & 0.02 & $H P R T$ & 0.02 & PPIA & 0.02 & $T B P$ & 0.02 \\
\hline & & 5 & RPLPO & 0.04 & $Y W H A Z$ & 0.04 & $A C T B$ & 0.02 & $Y W H A Z$ & 0.02 & $G A P D H$ & 0.03 & $Y W H A Z$ & 0.03 \\
\hline & & 6 & $R P L 13 A$ & 0.04 & RPL13A & 0.05 & $B 2 M$ & 0.03 & $T B P$ & 0.03 & $Y W H A Z$ & 0.03 & $G A P D H$ & 0 \\
\hline & & 7 & $Y W H A Z$ & 0.05 & $R P L P O$ & 0.06 & $R P L 13 A$ & 0.04 & $G A P D H$ & 0.03 & $G U S B$ & 0.04 & RPL13A & 0.03 \\
\hline & & 8 & $B 2 M$ & 0.06 & $T B P$ & 0.07 & $T B P$ & 0.04 & $B 2 M$ & 0.04 & RPL13A & 0.04 & GUSB & 0.03 \\
\hline & & 9 & $T B P$ & 0.07 & $B 2 M$ & 0.08 & $G U S B$ & 0.05 & RPL13A & 0.04 & $T B P$ & 0.04 & $A C T B$ & 0.04 \\
\hline & & 10 & $G U S B$ & 0.08 & $G U S B$ & 0.09 & RPLPO & 0.06 & $G U S B$ & 0.05 & $A C T B$ & 0.04 & RPLPO & 0.04 \\
\hline & & 11 & PPIA & 0.12 & PPIA & 0.14 & PPIA & 0.06 & $R P L P O$ & 0.06 & RPLPO & 0.05 & PPIA & 0.05 \\
\hline 21 & Induced & 1 & $G U S B$ & 0.02 & $A C T B$ & 0.02 & $G A P D H$ & 0.01 & $G A P D H$ & 0 & $B 2 M$ & 0.01 & $H B M S$ & 0 \\
\hline & & 2 & $Y W H A Z$ & 0.02 & $G U S B$ & 0.02 & $H B M S$ & 0.01 & $Y W H A Z$ & 0 & $Y W H A Z$ & 0.01 & $Y W H A Z$ & 0 \\
\hline & & 3 & $H B M S$ & 0.02 & $H B M S$ & 0.03 & $Y W H A Z$ & 0.01 & $H P R T$ & 0.01 & $A C T B$ & 0.01 & $B 2 M$ & 0.01 \\
\hline & & 4 & $A C T B$ & 0.03 & $Y W H A Z$ & 0.03 & $B 2 M$ & 0.01 & $B 2 M$ & 0.01 & $H B M S$ & 0.01 & $A C T B$ & 0.01 \\
\hline & & 5 & $T B P$ & 0.03 & $T B P$ & 0.03 & $H P R T$ & 0.01 & $A C T B$ & 0.02 & $G A P D H$ & 0.01 & PPIA & 0.01 \\
\hline & & 6 & $B 2 M$ & 0.04 & $B 2 M$ & 0.04 & $A C T B$ & 0.01 & $H B M S$ & 0.02 & $H P R T$ & 0.02 & $G A P D H$ & 0.01 \\
\hline & & 7 & $H P R T$ & 0.04 & $H P R T$ & 0.04 & $G U S B$ & 0.02 & $G U S B$ & 0.02 & PPIA & 0.02 & $H P R T$ & 0.02 \\
\hline & & 8 & $G A P D H$ & 0.06 & $G A P D H$ & 0.06 & PPIA & 0.02 & PPIA & 0.03 & $T B P$ & 0.02 & $T B P$ & 0.02 \\
\hline & & 9 & RPL13A & 0.07 & $R P L P O$ & 0.07 & $T B P$ & 0.03 & $T B P$ & 0.03 & $G U S B$ & 0.03 & $G U S B$ & 0.03 \\
\hline & & 10 & $R P L P O$ & 0.08 & RPL13A & 0.08 & RPL13A & 0.03 & RPL13A & 0.04 & RPL13A & 0.05 & RPL13A & 0.04 \\
\hline & & 11 & PPIA & 0.1 & PPIA & 0.11 & $R P L P O$ & 0.05 & RPLPO & 0.05 & RPLPO & 0.07 & $R P L P O$ & 0.07 \\
\hline All & & 1 & $T B P$ & 0.07 & $T B P$ & 0.07 & $H P R T$ & 0.03 & $H P R T$ & 0.03 & $B 2 M$ & 0.05 & $B 2 M$ & 0.05 \\
\hline & & 2 & $Y W H A Z$ & 0.07 & $Y W H A Z$ & 0.07 & $Y W H A Z$ & 0.03 & $Y W H A Z$ & 0.03 & $G U S B$ & 0.05 & $G U S B$ & 0.05 \\
\hline & & 3 & $H P R T$ & 0.08 & $H P R T$ & 0.08 & $H B M S$ & 0.04 & $H B M S$ & 0.04 & $T B P$ & 0.06 & $T B P$ & 0.05 \\
\hline & & 4 & $A C T B$ & 0.09 & $A C T B$ & 0.09 & $A C T B$ & 0.04 & $A C T B$ & 0.04 & $Y W H A Z$ & 0.07 & $Y W H A Z$ & 0.06 \\
\hline & & 5 & $G A P D H$ & 0.1 & $G A P D H$ & 0.1 & PPIA & 0.05 & $G A P D H$ & 0.04 & $H B M S$ & 0.07 & $H B M S$ & 0.07 \\
\hline & & 6 & $H B M S$ & 0.1 & $H B M S$ & 0.11 & $G A P D H$ & 0.05 & $T B P$ & 0.05 & PPIA & 0.07 & $H P R T$ & 0.07 \\
\hline & & 7 & $R P L P O$ & 0.12 & $R P L P O$ & 0.12 & $T B P$ & 0.06 & $B 2 M$ & 0.05 & $H P R T$ & 0.08 & $G A P D H$ & 0.08 \\
\hline & & 8 & $G U S B$ & 0.13 & $G U S B$ & 0.13 & $R P L 13 A$ & 0.06 & $G U S B$ & 0.06 & $G A P D H$ & 0.08 & $A C T B$ & 0.08 \\
\hline & & 9 & $B 2 M$ & 0.15 & $B 2 M$ & 0.15 & $G U S B$ & 0.07 & RPL13A & 0.06 & $A C T B$ & 0.08 & PPIA & 0.09 \\
\hline & & 10 & RPL13A & 0.16 & RPL13A & 0.17 & $B 2 M$ & 0.08 & PPIA & 0.07 & RPL13A & 0.1 & $R P L 13 A$ & 0.1 \\
\hline & & 11 & PPIA & 0.18 & PPIA & 0.18 & $R P L P O$ & 0.09 & $R P L P O$ & 0.08 & $R P L P O$ & 0.11 & $R P L P O$ & 0.11 \\
\hline
\end{tabular}




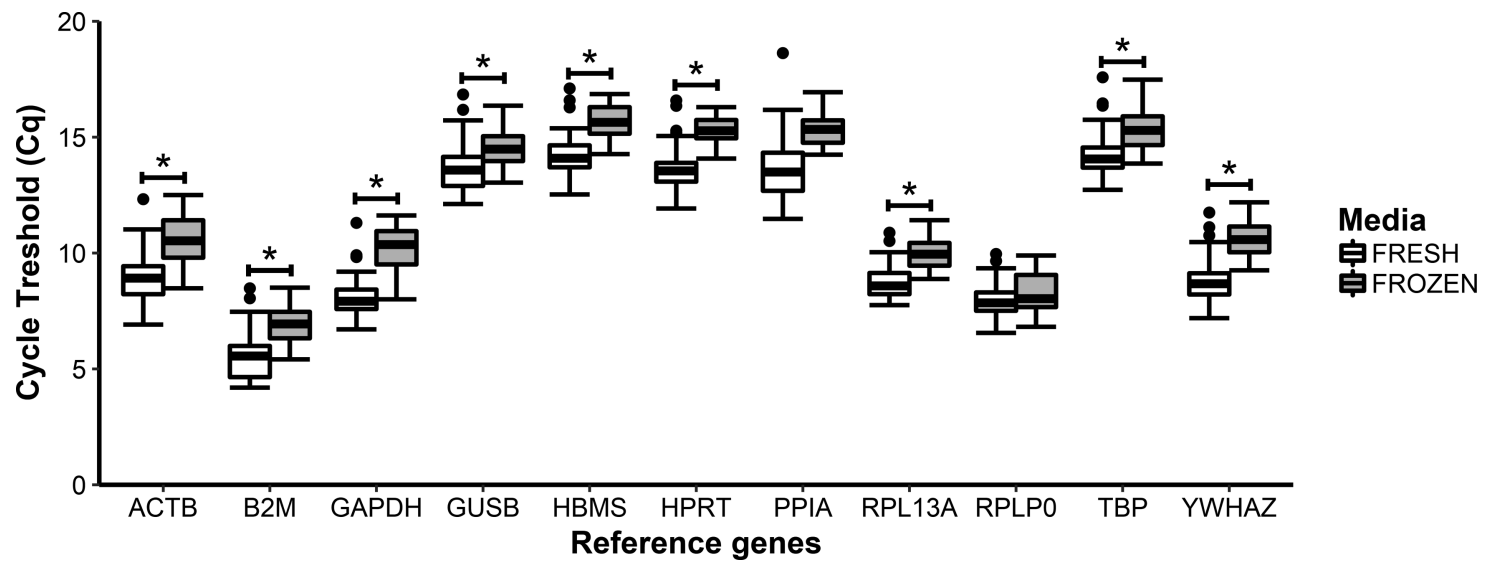

FIG. 2. Box and whisker plots of the Cq values for the $11 \mathrm{RGs}$ assessed in freshly isolated ASCs expanded in FBS and previously cryopreserved ASCs expanded in FBS. Boxes extend from the first to third quartiles with the median shown as the solid black line intersecting the box; whiskers extend to the minimum and maximum values that lie within $1.5 \times$ the IQR. Data points beyond the whiskers represent outliers. The sample size is $n=36$ and represents four biological replicates at nine time points at different differentiation states (day 0, days 1 control and induced, day 7 control and indicated, day 14 control and induced and day $21 \mathrm{control}$ and induced). $*$ Statistical significance $p<0.05$. ASC, adipose-derived stromal cell; FBS, fetal bovine serum.

standards and the cells should retain their unique cellspecific characteristics. ${ }^{28}$ One of these characteristics is the ability to differentiate into adipocytes, which also provides a valuable tool for understanding the process of adipogenesis in obesity research. ${ }^{17}$

qPCR is used to measure the effects of experimental conditions on gene expression. Guidelines were published to aid researchers in experimental design and quality assurance to produce reproducible data ${ }^{25,29}$ : these include the selection of stable RGs. RGs should remain constant in all cells and not change under experimental conditions. Some studies make use of one RG for normalization, which may affect interpretation of results. The use of a geometric mean of multiple RGs for normalization is preferable..$^{2,30,31}$

Many platforms that assess RG stability are available and they use different algorithms to rank the most stable RGs in a given panel. ${ }^{25,26,32}$ Several studies have compared the platforms with one another and have mostly reported that the results are similar between them. ${ }^{33,34}$ The most commonly and widely used platform is geNorm, ${ }^{25}$ which requires the input data to be converted from raw $\mathrm{Cq}$ values into relative expression values using the equation $E^{-\Delta \mathrm{Cq}}$. A number of studies assume that efficiencies are $100 \%$ and set $E=2$, whereas other studies calculate efficiencies from the standard curves for each of the RGs. The use of different efficiencies has been shown to impact the ranking of RG stability, ${ }^{33}$ especially when the efficiency deviates from its ideal ( $E=2,100 \%$ efficiency). By using SE, technical variation is taken into consideration and provides a more accurate result. In this study, we observed that the $E=2$ and SE methods yielded similar results with regard to position changes in the rankings of RGs in the different groups.

The choice of sample grouping or testing subsets differs in the literature. ${ }^{23,35-37}$ Some studies group all of their samples, regardless of the nature of the experiment, and report on the overall RG stability. In a study by Fink et al., ${ }^{23}$ all samples for each passage; hypoxic treatment; and adipogenic, osteogenic, and chondrogenic differentiation were grouped together and the authors reported on the overall stability of the RGs. Other studies have separated samples into experimental groups or subsets. In a study by Li et al., ${ }^{35}$ samples were grouped into separate developmental stages of the different parts of the celery plant; they then combined the development stages and looked at the overall stability in the different parts of the celery plant; and lastly, all samples were combined into one group and overall stability within the celery plant was described.

In our study, we grouped our samples according to the assumptions we make (Table 2) in our adipogenic differentiation assay and how the different experimental conditions affect the adipogenic capacity of ASCs. In our adipogenic differentiation assay, ${ }^{9,17}$ we have investigated changes in gene expression for 21 days. We measured gene expression on different days by normalizing our induced samples to our control samples and then compare the fold changes in gene expression at the different time points. To accurately report on changes in fold expression, we assumed that the RGs are stable and that they do not change significantly between days in either the control or the induced samples. Another assumption we made is that the experimental conditions (medium choice and cryopreservation status) do not affect RG stability. In this study, we have tested these assumptions by measuring the effect that different experimental conditions have on RG stability using both the $E=2$ and SE methods. More specifically, we examined RG stability (1) at the different time points in the differentiation assay, (2) between control and induced samples, (3) between cryopreservation states, and (4) between different media used for expansion purposes.

Irrespective of the efficiency method or experimental grouping used, we found that all the stability values were $<0.5$ (Tables 3 and 4), suggesting that all 11 RGs can be considered to be stable, although some ranked better than others. Furthermore, when considering the optimal number of RGs to be used for normalization, all the RG pairwise variation values were $<0.15$ (Supplementary Tables S3 and S4), 
Table 4. Specific and 100\% Efficiency Reference Gene Stability Rankings and Values For the 11 Reference Genes in the Fresh FBS and Frozen FBS Groups and in the Frozen FBS and Frozen HPL Groups at Each Time Point, in Induced and Control Samples

\begin{tabular}{|c|c|c|c|c|c|c|c|c|c|c|}
\hline \multirow[b]{3}{*}{ Day } & \multirow[b]{3}{*}{ Differentiation } & \multirow[b]{3}{*}{ Rank } & \multicolumn{4}{|c|}{ Fresh FBS vs. frozen FBS } & \multicolumn{4}{|c|}{ Frozen FBS vs. frozen $H P L$} \\
\hline & & & \multicolumn{2}{|c|}{$100 \%$ efficiency } & \multicolumn{2}{|c|}{ Specific efficiency } & \multicolumn{2}{|c|}{$100 \%$ efficiency } & \multicolumn{2}{|c|}{ Specific efficiency } \\
\hline & & & $R G$ & $M$ & $R G$ & $M$ & $R G$ & $M$ & $R G$ & $M$ \\
\hline \multirow[t]{11}{*}{0} & \multirow[t]{11}{*}{ Control } & 1 & $G A P D H$ & 0.03 & $A C T B$ & 0.03 & $G A P D H$ & 0.02 & $G U S B$ & 0.04 \\
\hline & & 2 & $Y W H A Z$ & 0.03 & $H P R T$ & 0.03 & $Y W H A Z$ & 0.02 & $R P L 13 A$ & 0.04 \\
\hline & & 3 & HPRT & 0.04 & $Y W H A Z$ & 0.04 & $H B M S$ & 0.04 & $B 2 M$ & 0.05 \\
\hline & & 4 & $B 2 M$ & 0.05 & $H B M S$ & 0.05 & PPIA & 0.07 & $T B P$ & 0.06 \\
\hline & & 5 & $A C T B$ & 0.05 & $G A P D H$ & 0.05 & $T B P$ & 0.09 & $R P L P O$ & 0.06 \\
\hline & & 6 & $H B M S$ & 0.06 & $B 2 M$ & 0.06 & $R P L P O$ & 0.1 & $G A P D H$ & 0.09 \\
\hline & & 7 & $T B P$ & 0.07 & $T B P$ & 0.06 & $R P L 13 A$ & 0.1 & $Y W H A Z$ & 0.1 \\
\hline & & 8 & $R P L 13 A$ & 0.07 & $G U S B$ & 0.07 & $B 2 M$ & 0.11 & $H B M S$ & 0.1 \\
\hline & & 9 & $G U S B$ & 0.08 & $R P L 13 A$ & 0.07 & $G U S B$ & 0.11 & PPIA & 0.11 \\
\hline & & 10 & PPIA & 0.08 & $R P L P O$ & 0.08 & $H P R T$ & 0.12 & $H P R T$ & 0.12 \\
\hline & & 11 & $R P L P O$ & 0.1 & PPIA & 0.1 & $A C T B$ & 0.13 & $A C T B$ & 0.13 \\
\hline \multirow[t]{11}{*}{1} & \multirow[t]{11}{*}{ Control } & 1 & $G A P D H$ & 0.07 & $G A P D H$ & 0.07 & $H P R T$ & 0.02 & $H P R T$ & 0.03 \\
\hline & & 2 & $T B P$ & 0.07 & $H P R T$ & 0.07 & $Y W H A Z$ & 0.02 & $Y W H A Z$ & 0.03 \\
\hline & & 3 & $H B M S$ & 0.07 & $H B M S$ & 0.07 & $R P L 13 A$ & 0.03 & $R P L 13 A$ & 0.03 \\
\hline & & 4 & $H P R T$ & 0.08 & $T B P$ & 0.08 & $B 2 M$ & 0.04 & $B 2 M$ & 0.04 \\
\hline & & 5 & $Y W H A Z$ & 0.09 & $Y W H A Z$ & 0.09 & $H B M S$ & 0.04 & $H B M S$ & 0.04 \\
\hline & & 6 & $A C T B$ & 0.09 & $A C T B$ & 0.1 & PPIA & 0.05 & $T B P$ & 0.05 \\
\hline & & 7 & $B 2 M$ & 0.11 & $B 2 M$ & 0.11 & $T B P$ & 0.05 & $R P L P O$ & 0.05 \\
\hline & & 8 & $R P L 13 A$ & 0.12 & $R P L 13 A$ & 0.12 & $G A P D H$ & 0.06 & $G U S B$ & 0.05 \\
\hline & & 9 & $G U S B$ & 0.13 & $G U S B$ & 0.13 & $A C T B$ & 0.07 & $G A P D H$ & 0.06 \\
\hline & & 10 & $R P L P O$ & 0.14 & $R P L P O$ & 0.15 & $R P L P O$ & 0.07 & $A C T B$ & 0.07 \\
\hline & & 11 & PPIA & 0.16 & PPIA & 0.17 & $G U S B$ & 0.08 & PPIA & 0.07 \\
\hline \multirow[t]{11}{*}{1} & \multirow[t]{11}{*}{ Induced } & 1 & $H P R T$ & 0.08 & $H P R T$ & 0.03 & $H P R T$ & 0.02 & $H P R T$ & 0.01 \\
\hline & & 2 & $Y W H A Z$ & 0.08 & $Y W H A Z$ & 0.03 & $Y W H A Z$ & 0.02 & $Y W H A Z$ & 0.01 \\
\hline & & 3 & $T B P$ & 0.09 & $B 2 M$ & 0.05 & $A C T B$ & 0.03 & $A C T B$ & 0.03 \\
\hline & & 4 & $A C T B$ & 0.1 & $G U S B$ & 0.06 & $H B M S$ & 0.04 & $H B M S$ & 0.03 \\
\hline & & 5 & $H B M S$ & 0.12 & $T B P$ & 0.07 & $R P L 13 A$ & 0.04 & $R P L 13 A$ & 0.04 \\
\hline & & 6 & $G A P D H$ & 0.12 & $A C T B$ & 0.08 & $B 2 M$ & 0.05 & $B 2 M$ & 0.04 \\
\hline & & 7 & $B 2 M$ & 0.13 & $R P L 13 A$ & 0.1 & $T B P$ & 0.06 & $T B P$ & 0.05 \\
\hline & & 8 & $G U S B$ & 0.14 & $G A P D H$ & 0.11 & PPIA & 0.07 & $G U S B$ & 0.06 \\
\hline & & 9 & $R P L 13 A$ & 0.14 & $H B M S$ & 0.12 & $G U S B$ & 0.08 & PPIA & 0.07 \\
\hline & & 10 & $R P L P O$ & 0.15 & PPIA & 0.12 & $G A P D H$ & 0.09 & $G A P D H$ & 0.09 \\
\hline & & 11 & PPIA & 0.17 & $R P L P O$ & 0.13 & $R P L P O$ & 0.1 & $R P L P O$ & 0.1 \\
\hline 7 & Control & 1 & $G U S B$ & 0.05 & $G U S B$ & 0.05 & $H B M S$ & 0.03 & $H B M S$ & 0.03 \\
\hline & & 2 & $R P L 13 A$ & 0.05 & $H B M S$ & 0.05 & $Y W H A Z$ & 0.03 & $H P R T$ & 0.03 \\
\hline & & 3 & $H B M S$ & 0.06 & $T B P$ & 0.06 & $H P R T$ & 0.03 & $Y W H A Z$ & 0.03 \\
\hline & & 4 & $T B P$ & 0.07 & $Y W H A Z$ & 0.06 & PPIA & 0.04 & $G A P D H$ & 0.04 \\
\hline & & 5 & $Y W H A Z$ & 0.08 & $H P R T$ & 0.07 & $G A P D H$ & 0.05 & $T B P$ & 0.04 \\
\hline & & 6 & $G A P D H$ & 0.08 & $G A P D H$ & 0.08 & $R P L 13 A$ & 0.06 & $R P L 13 A$ & 0.05 \\
\hline & & 7 & $H P R T$ & 0.09 & $B 2 M$ & 0.08 & $T B P$ & 0.06 & PPIA & 0.05 \\
\hline & & 8 & $B 2 M$ & 0.09 & $R P L 13 A$ & 0.09 & $A C T B$ & 0.07 & $G U S B$ & 0.06 \\
\hline & & 9 & $A C T B$ & 0.1 & $A C T B$ & 0.09 & $G U S B$ & 0.08 & $B 2 M$ & 0.06 \\
\hline & & 10 & $R P L P O$ & 0.11 & $R P L P O$ & 0.1 & $B 2 M$ & 0.08 & $A C T B$ & 0.07 \\
\hline & & 11 & PPIA & 0.13 & PPIA & 0.12 & $R P L P O$ & 0.09 & $R P L P O$ & 0.08 \\
\hline 7 & Induced & 1 & $A C T B$ & 0.02 & $A C T B$ & 0.02 & $H P R T$ & 0.03 & $B 2 M$ & 0.03 \\
\hline & & 2 & $Y W H A Z$ & 0.02 & $Y W H A Z$ & 0.02 & $Y W H A Z$ & 0.03 & $G U S B$ & 0.03 \\
\hline & & 3 & PPIA & 0.03 & $G A P D H$ & 0.04 & $H B M S$ & 0.04 & $Y W H A Z$ & 0.04 \\
\hline & & 4 & $G A P D H$ & 0.04 & HPRT & 0.05 & $B 2 M$ & 0.04 & $H B M S$ & 0.04 \\
\hline & & 5 & HPRT & 0.04 & $H B M S$ & 0.05 & $G U S B$ & 0.05 & $H P R T$ & 0.05 \\
\hline & & 6 & $T B P$ & 0.05 & $B 2 M$ & 0.06 & PPIA & 0.05 & $T B P$ & 0.05 \\
\hline & & 7 & $B 2 M$ & 0.06 & $T B P$ & 0.07 & $T B P$ & 0.06 & $A C T B$ & 0.06 \\
\hline & & 8 & $H B M S$ & 0.06 & PPIA & 0.08 & $A C T B$ & 0.06 & $G A P D H$ & 0.07 \\
\hline & & 9 & $G U S B$ & 0.07 & $G U S B$ & 0.08 & $G A P D H$ & 0.07 & PPIA & 0.08 \\
\hline & & 10 & $R P L 13 A$ & 0.08 & $R P L 13 A$ & 0.09 & $R P L 13 A$ & 0.08 & RPL13A & 0.09 \\
\hline & & 11 & $R P L P O$ & 0.09 & $R P L P O$ & 0.1 & $R P L P O$ & 0.1 & $R P L P O$ & 0.11 \\
\hline
\end{tabular}


TABle 4. (Continued)

\begin{tabular}{|c|c|c|c|c|c|c|c|c|c|c|}
\hline \multirow[b]{3}{*}{ Day } & \multirow[b]{3}{*}{ Differentiation } & \multirow[b]{3}{*}{ Rank } & \multicolumn{4}{|c|}{ Fresh FBS vs. frozen FBS } & \multicolumn{4}{|c|}{ Frozen FBS vs. frozen $H P L$} \\
\hline & & & \multicolumn{2}{|c|}{$100 \%$ efficiency } & \multicolumn{2}{|c|}{ Specific efficiency } & \multicolumn{2}{|c|}{$100 \%$ efficiency } & \multicolumn{2}{|c|}{ Specific efficiency } \\
\hline & & & $R G$ & $M$ & $R G$ & $M$ & $R G$ & $M$ & $R G$ & $M$ \\
\hline \multirow[t]{11}{*}{14} & \multirow[t]{11}{*}{ Control } & 1 & $H B M S$ & 0.03 & $A C T B$ & 0.04 & $H B M S$ & 0.02 & $H B M S$ & 0.01 \\
\hline & & 2 & $Y W H A Z$ & 0.03 & $Y W H A Z$ & 0.04 & $H P R T$ & 0.02 & $H P R T$ & 0.01 \\
\hline & & 3 & $A C T B$ & 0.04 & $H B M S$ & 0.04 & $Y W H A Z$ & 0.02 & $Y W H A Z$ & 0.02 \\
\hline & & 4 & $H P R T$ & 0.04 & $H P R T$ & 0.04 & $G A P D H$ & 0.03 & $G A P D H$ & 0.03 \\
\hline & & 5 & $R P L 13 A$ & 0.04 & $T B P$ & 0.04 & PPIA & 0.04 & $A C T B$ & 0.04 \\
\hline & & 6 & $T B P$ & 0.05 & RPL13A & 0.05 & $A C T B$ & 0.05 & $T B P$ & 0.04 \\
\hline & & 7 & $G A P D H$ & 0.06 & $G U S B$ & 0.06 & $T B P$ & 0.05 & $R P L 13 A$ & 0.05 \\
\hline & & 8 & $G U S B$ & 0.07 & $G A P D H$ & 0.06 & $R P L 13 A$ & 0.06 & $R P L P O$ & 0.06 \\
\hline & & 9 & $B 2 M$ & 0.08 & $R P L P O$ & 0.07 & $R P L P O$ & 0.06 & $G U S B$ & 0.06 \\
\hline & & 10 & PPIA & 0.09 & $B 2 M$ & 0.09 & $G U S B$ & 0.07 & $B 2 M$ & 0.07 \\
\hline & & 11 & $R P L P O$ & 0.09 & PPIA & 0.1 & $B 2 M$ & 0.08 & PPIA & 0.08 \\
\hline \multirow[t]{11}{*}{14} & \multirow[t]{11}{*}{ Induced } & 1 & $B 2 M$ & 0.02 & $B 2 M$ & 0.03 & $H B M S$ & 0.01 & $H B M S$ & 0.01 \\
\hline & & 2 & $H P R T$ & 0.02 & $Y W H A Z$ & 0.03 & $H P R T$ & 0.01 & $Y W H A Z$ & 0.01 \\
\hline & & 3 & $Y W H A Z$ & 0.02 & $G U S B$ & 0.03 & $Y W H A Z$ & 0.03 & GAPDH & 0.02 \\
\hline & & 4 & $G U S B$ & 0.03 & $T B P$ & 0.04 & $G A P D H$ & 0.03 & $A C T B$ & 0.02 \\
\hline & & 5 & $T B P$ & 0.03 & $H P R T$ & 0.04 & PPIA & 0.03 & $G U S B$ & 0.03 \\
\hline & & 6 & $G A P D H$ & 0.04 & $G A P D H$ & 0.04 & $A C T B$ & 0.03 & $H P R T$ & 0.03 \\
\hline & & 7 & $H B M S$ & 0.04 & $H B M S$ & 0.05 & $T B P$ & 0.04 & $T B P$ & 0.04 \\
\hline & & 8 & $A C T B$ & 0.05 & $A C T B$ & 0.05 & $R P L 13 A$ & 0.04 & $B 2 M$ & 0.04 \\
\hline & & 9 & $R P L P O$ & 0.06 & $R P L P O$ & 0.06 & $R P L P O$ & 0.05 & PPIA & 0.05 \\
\hline & & 10 & PPIA & 0.07 & PPIA & 0.08 & $G U S B$ & 0.06 & RPL13A & 0.06 \\
\hline & & 11 & $R P L 13 A$ & 0.09 & $R P L 13 A$ & 0.1 & $B 2 M$ & 0.09 & $R P L P O$ & 0.08 \\
\hline \multirow[t]{11}{*}{21} & \multirow[t]{11}{*}{ Control } & 1 & $A C T B$ & 0.02 & $A C T B$ & 0.03 & $H B M S$ & 0.01 & $H B M S$ & 0.01 \\
\hline & & 2 & $H B M S$ & 0.02 & $H B M S$ & 0.03 & $H P R T$ & 0.01 & $H P R T$ & 0.01 \\
\hline & & 3 & HPRT & 0.03 & $H P R T$ & 0.04 & PPIA & 0.03 & $Y W H A Z$ & 0.03 \\
\hline & & 4 & $Y W H A Z$ & 0.05 & $Y W H A Z$ & 0.05 & $Y W H A Z$ & 0.04 & $T B P$ & 0.03 \\
\hline & & 5 & $G A P D H$ & 0.06 & $T B P$ & 0.06 & $G A P D H$ & 0.04 & $G A P D H$ & 0.04 \\
\hline & & 6 & $T B P$ & 0.07 & $G A P D H$ & 0.07 & $T B P$ & 0.05 & $A C T B$ & 0.04 \\
\hline & & 7 & $R P L 13 A$ & 0.08 & $R P L 13 A$ & 0.08 & $R P L 13 A$ & 0.05 & PPIA & 0.05 \\
\hline & & 8 & $R P L P O$ & 0.08 & $R P L P O$ & 0.09 & $A C T B$ & 0.06 & RPL13A & 0.06 \\
\hline & & 9 & $G U S B$ & 0.1 & $G U S B$ & 0.1 & $R P L P O$ & 0.07 & $R P L P O$ & 0.07 \\
\hline & & 10 & $B 2 M$ & 0.11 & $B 2 M$ & 0.12 & $G U S B$ & 0.08 & $G U S B$ & 0.07 \\
\hline & & 11 & PPIA & 0.13 & PPIA & 0.14 & $B 2 M$ & 0.09 & $B 2 M$ & 0.08 \\
\hline 21 & Induced & 1 & $A C T B$ & 0.03 & $A C T B$ & 0.03 & $B 2 M$ & 0.02 & $B 2 M$ & 0.02 \\
\hline & & 2 & $H B M S$ & 0.03 & $H B M S$ & 0.03 & $H B M S$ & 0.02 & $Y W H A Z$ & 0.02 \\
\hline & & 3 & $G U S B$ & 0.03 & $B 2 M$ & 0.03 & $Y W H A Z$ & 0.02 & $H B M S$ & 0.02 \\
\hline & & 4 & $Y W H A Z$ & 0.03 & $G U S B$ & 0.03 & $H P R T$ & 0.02 & $H P R T$ & 0.02 \\
\hline & & 5 & $B 2 M$ & 0.04 & $Y W H A Z$ & 0.04 & $G A P D H$ & 0.03 & PPIA & 0.02 \\
\hline & & 6 & HPRT & 0.04 & $T B P$ & 0.04 & PPIA & 0.03 & $G A P D H$ & 0.03 \\
\hline & & 7 & $T B P$ & 0.05 & $H P R T$ & 0.05 & $A C T B$ & 0.03 & $A C T B$ & 0.03 \\
\hline & & 8 & $G A P D H$ & 0.06 & $G A P D H$ & 0.06 & $T B P$ & 0.03 & $T B P$ & 0.03 \\
\hline & & 9 & PPIA & 0.07 & $R P L P O$ & 0.07 & $G U S B$ & 0.04 & $G U S B$ & 0.04 \\
\hline & & 10 & $R P L P O$ & 0.09 & $R P L 13 A$ & 0.09 & $R P L 13 A$ & 0.05 & $R P L 13 A$ & 0.04 \\
\hline & & 11 & $R P L 13 A$ & 0.1 & $P P I A$ & 0.11 & $R P L P O$ & 0.07 & $R P L P O$ & 0.06 \\
\hline All & & 1 & $H P R T$ & 0.06 & $H P R T$ & 0.06 & $H B M S$ & 0.04 & $H P R T$ & 0.05 \\
\hline & & 2 & $Y W H A Z$ & 0.06 & $Y W H A Z$ & 0.06 & HPRT & 0.04 & $Y W H A Z$ & 0.05 \\
\hline & & 3 & $A C T B$ & 0.07 & $T B P$ & 0.07 & PPIA & 0.05 & $H B M S$ & 0.05 \\
\hline & & 4 & $T B P$ & 0.08 & $A C T B$ & 0.07 & $G A P D H$ & 0.06 & $T B P$ & 0.06 \\
\hline & & 5 & $G A P D H$ & 0.08 & $H B M S$ & 0.08 & $Y W H A Z$ & 0.07 & $G A P D H$ & 0.07 \\
\hline & & 6 & $H B M S$ & 0.09 & $G A P D H$ & 0.09 & $A C T B$ & 0.07 & $A C T B$ & 0.07 \\
\hline & & 7 & $G U S B$ & 0.1 & $G U S B$ & 0.1 & $T B P$ & 0.08 & PPIA & 0.08 \\
\hline & & 8 & $B 2 M$ & 0.12 & $R P L P O$ & 0.12 & $R P L 13 A$ & 0.09 & $B 2 M$ & 0.09 \\
\hline & & 9 & PPIA & 0.13 & $B 2 M$ & 0.13 & $G U S B$ & 0.09 & $G U S B$ & 0.09 \\
\hline & & 10 & $R P L P O$ & 0.14 & RPL13A & 0.14 & $B 2 M$ & 0.1 & RPL13A & 0.1 \\
\hline & & 11 & $R P L 13 A$ & 0.15 & PPIA & 0.15 & RPLPO & 0.11 & $R P L P O$ & 0.11 \\
\hline
\end{tabular}




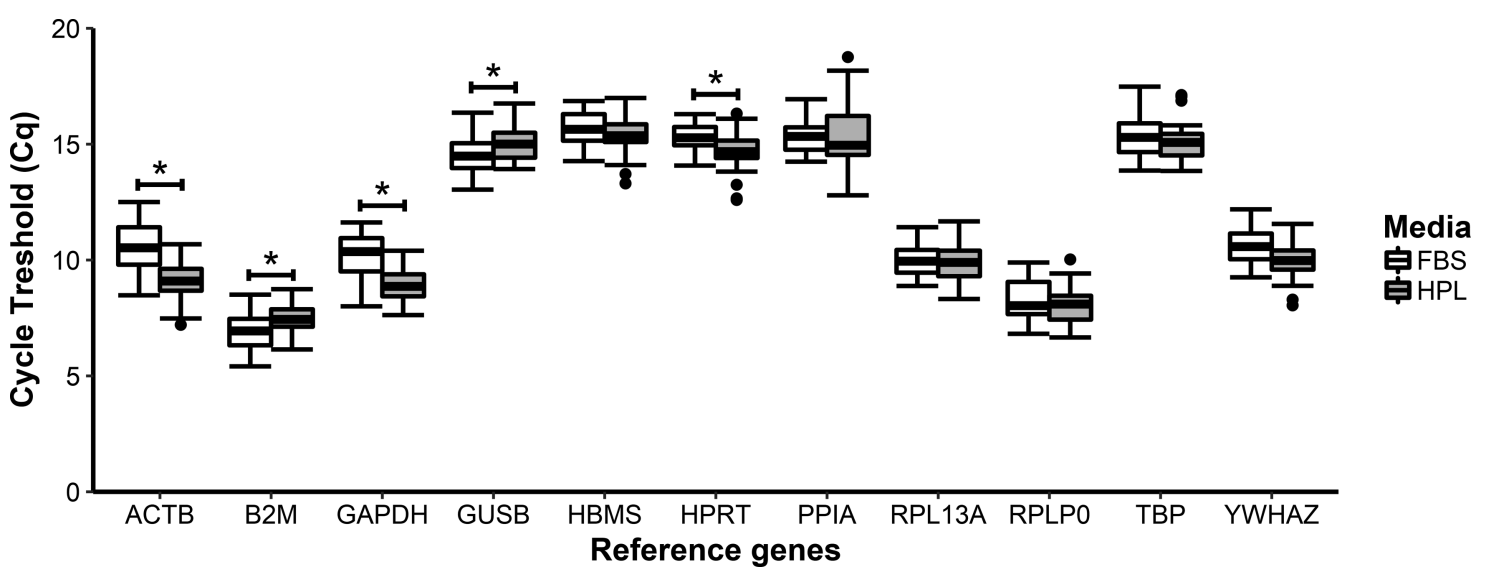

FIG. 3. Box and whisker plots of $\mathrm{Cq}$ values for the $11 \mathrm{RG}$ assessed in previously cryopreserved ASCs expanded in FBS and previously cryopreserved ASCs expanded in pHPL. Boxes extend from the first to third quartiles with the median shown as the solid black line intersecting the box; the whiskers extend to the minimum and maximum values that lie within $1.5 \times$ the IQR. Data points beyond the whiskers represent outliers. The sample size is $n=36$ and represents four biological replicates at nine time points at different differentiation states (day 0, days 1 control and induced, day 7 control and indicated, day 14 control and induced, and day 21 control and induced). *Statistical significance $p<0.05$.

suggesting that only two RGs are required for normalization and the addition of more RGs would provide no significant improvement. However, the use of more stable RGs can reduce variations in expression levels and is recommended. ${ }^{23}$

When compared between days and between control and induced samples, we found that only a few RGs had no change in $\mathrm{Cq}$ values in the different experimental groups (Supplementary Figs. S5-S7 and Supplementary Table S2). This corresponds with other studies where the effect of time on culture and treatment affects RG stability. ${ }^{38-40}$ When all the time points and control and induced samples were grouped together, the most stable RGs in the fresh FBS group were TBP, YWHAZ, and HPRT; those in the frozen FBS group were $H P R T, Y W H A Z$, and $H B M S$; and those in the frozen HPL group were $B 2 M, T B P$, and $G U S B$.

Conflicting data have been reported on the effect of cryopreservation on the adipogenic capacity of ASCs. James et al. ${ }^{41}$ found that cryopreservation negatively affects adipogenic potential, whereas Yong et al. ${ }^{42}$ found that cryopreservation did not affect adipogenic potential in ASCs. Both of these studies made use of GAPDH for normalization, but neither indicated how the stability of $G A P D H$ was measured. We, therefore, measured the effect of cryopreservation on RG stability by comparing freshly isolated ASCs with previously frozen ASCs, both expanded in FBS. We grouped all the control and induced samples into a fresh FBS or a frozen FBS group. When comparing RGs between the two groups, we found no significant changes in PPIA or RPLPO $\mathrm{Cq}$ values (Fig. 2), even though they consistently ranked as the least stable RGs for all time points in the control and induced samples using both efficiency methods (Table 4). The lack of significance could be explained by the variability seen in the $\mathrm{Cq}$ values of PPIA in the fresh FBS group and of $R P L P O$ in the frozen FBS group, whereas the variability is taken into consideration by the algorithm of the geNorm software and negatively affects their ranking. In contrast, geNorm ranked HPRT, YWHAZ, ACTB, and TBP as the most stable RGs, where HPRT and $Y W H A Z$ were ranked identically for both the efficiency methods, and $A C T B$ and TBP switched positions depending on the efficiency method used.
The use of pHPL for ASC expansion has numerous advantages over FBS. ${ }^{43-45}$ Most studies have shown that pHPL has little or no effect on the adipogenic potential of ASCs when compared with FBS. ${ }^{46,47}$ In our study, freshly isolated ASCs expanded in $10 \%$ pHPL detached after a few days in the differentiation assay; as a result we used cryopreserved ASCs expanded in 5\% pHPL. The detachment of freshly isolated ASCs expanded in pHPL was also reported by Blande et $a .^{48}$ In their study, the authors showed that cryopreserved ASCs expanded in a reduced concentration of pHPL had the ability to differentiate into adipocytes as confirmed by histochemical staining; although this was qualitative evidence, in our study we have used a quantitative approach. Therefore, we investigated whether RG stability differed when the medium was supplemented with either FBS or pHPL in the same cryopreservation state. We grouped control and induced samples of the previously frozen ASCs into either a frozen FBS or a frozen HPL group. When compared, the $\mathrm{Cq}$ values of $H B M S$, PPIA, RPL13A, RPLPO, TBP, and YWHAZ were not significantly different between the frozen FBS and frozen pHPL groups (Fig. 3). When stability was ranked, HPRT and HBMS were in the top three stable RGs with minor position changes depending on the efficiency method used (Table 4). YWHAZ was ranked as one of the most stable genes for the SE method, whereas PPIA was ranked as one of the most stable RGs when $E=2$ was used. $R P L P O$ ranked as the least stable when all of the control and induced samples were grouped together, irrespective of the efficiency method used.

When considering the entire study, PPIA had the lowest $R^{2}$ value, the smallest $E$ value, the greatest variability, and regularly appeared toward the bottom of the stability rankings, except when the frozen FBS and frozen HPL groups were compared. These findings are in contrast to those of Fink et al. who found that PPIA was stable during adipogenesis and Tratwal et al., who found PPIA to be stable during cell expansion. ${ }^{8,23}$ In our study, in the fresh FBS groups on the different days in control and induced samples (Table 3), PPIA consistently ranked as the least stable RG using both efficiency methods; however, in the frozen FBS 
and frozen pHPL groups on different days in control and induced samples, PPIA showed major position changes in both efficiency methods. Based on these findings, we concluded that PPIA should not be considered as a stable RG and consequently should not be used in further studies.

It is well known that primary cells such as ASCs display both inter- and intrapatient variability before and after differentiation. ${ }^{49}$ These variations could affect some of the most important adipocyte functions such as lipid formation, insulin sensitivity, and adipokine function, all of which can affect RG stability. This study did not access these possible differences and should be considered if functional studies are being performed. Furthermore, to prevent experimental bias in our comparisons, the experimental design was identical between all of the experimental conditions.

In this study we did not find any one specific RG that consistently ranked as the most stable in all experimental groups. However, there were RGs that appeared more regularly in the higher rankings. We further found that RG stability differed between days, differentiation status, cryopreservation status, and the expansion medium used. Similar findings were established by Ferguson et al. when comparing RG stability in 3T3-L1 adipocytes under different experimental conditions. ${ }^{50}$

\section{Conclusions}

We suggest that the use of RGs for normalization should be selected on the basis of the experiment being performed. For adipogenic differentiation for a 21 day induction period for a single experimental condition (control vs. induced), we suggest using RGs specific to the different groups being assessed: TBP, YWHAZ, HPRT, and ACTB for freshly isolated ASCs expanded in FBS; HPRT, YWHAZ, $H B M S$, and $A C T B$ for previously frozen ASCs expanded in FBS; and $B 2 M, G U S B, T B P$, and $Y W H A Z$ for previously frozen ASCs expanded in pHPL. When introducing more than one experimental condition during adipogenic differentiation (e.g., fresh vs thawed ASCs expanded in FBS or pHPL), we propose using HPRT, YWHAZ, ACTB, and $T B P$ for comparing fresh versus cryopreserved cells, and $H B M S, H P R T, Y W H A Z$, and TBP for the comparison of different media.

\section{Declarations}

\section{Ethics approval and consent to participate}

Informed consent was obtained before the isolation procedure from lipoaspirate, and approval for the study was granted by the University of Pretoria Health Sciences Research Ethics Committee (approval number 421/2013), as well as the Human Research Committee, SANBS, South Africa (2013/17).

\section{Authors' Contributions}

C.D. participated in all the experimental work, analysis thereof, and drafted the article. M.S.P. conceived the overall project, participated in the project design and coordination, raised the funding, and edited the article. All authors have read and approved the final article.

\section{Acknowledgments}

We thank Amy Hamilton (Fluidigm) for assisting in the experiments and analysis performed on the Biomark, Prof. P. Coetzee (Head of Plastic Surgery, Steve Biko Academic Hospital) and Dr. D. Hoffman (private practice) for the lipoaspirate samples, Stephen Marrs and Heamotec (South Africa) for the consumables, and SANBS for the blood products provided.

This study was supported by a grant from the South African Medical Research Council (SAMRC) University Flagship Project SAMRC-RFA-UFSP-01-2013/STEM CELLS, the SAMRC Extramural Unit for Stem Cell Research and Therapy, and the Institute for Cellular and Molecular Medicine of the University of Pretoria.

\section{Disclosure Statement}

The authors declare that they have no competing interests.

\section{Supplementary Material}

Supplementary Data

Supplementary Figure S1

Supplementary Figure S2

Supplementary Figure S3

Supplementary Figure S4

Supplementary Figure S5

Supplementary Figure S6

Supplementary Figure S7

Supplementary Table S1

Supplementary Table S2

Supplementary Table S3

Supplementary Table S4

\section{References}

1. Gimble, J.M., Ray, S.P., Zanata, F., et al. Adipose derived cells and tissues for regenerative medicine. ACS Biomater Sci Eng 3, 1477, 2016.

2. Camilleri, E.T., Gustafson, M.P., Dudakovic, A., et al. Identification and validation of multiple cell surface markers of clinical-grade adipose- derived mesenchymal stromal cells as novel release criteria for good manufacturing practice-compliant production. Stem Cell Res Ther 7, 107, 2016.

3. Toyserkani, N.M., Jørgensen, M.G., Tabatabaeifar, S., Harken Jensen, C., Sheikh, S.P., Sørensen, J.A. Concise Review: a Safety Assessment of Adipose- Derived Cell Therapy in Clinical Trials: a Systematic Review of Reported Adverse Events. Stem Cells Transl Med 6, 1786, 2017.

4. Giancola, R., Bonfini, T., Iacone, A. Cell therapy: cGMP facilities and manufacturing. Muscles Ligaments Tendons J 2, 243, 2012.

5. Riis, S., Zachar, V., Boucher, S., Vemuri, M.C., Pennisi, C.P., Fink, T. Critical steps in the isolation and expansion of adipose-derived stem cells for translational therapy. Expert Rev Mol Med 17(e11), 1, 2015.

6. Shahdadfar, A., Frønsdal, K., Haug, T., Reinholt, F.P., Brinchmann, J.E. In vitro expansion of human mesenchymal stem cells: choice of serum is a determinant of cell proliferation, differentiation, gene expression, and transcriptome stability. Stem Cells 23, 1357, 2005. 
7. Oliveira, P.H., Boura, J.S., Abecasis, M.M., Gimble, J.M., da Silva, C.L., Cabral JMS. Impact of hypoxia and longterm cultivation on the genomic stability and mitochondrial performance of ex vivo expanded human stem/stromal cells. Stem Cell Res 9, 225, 2012.

8. Tratwal, J., Follin, B., Ekblond, A., Kastrup, J., HaackSørensen, M. Identification of a common reference gene pair for qPCR in human mesenchymal stromal cells from different tissue sources treated with VEGF. BMC Mol Biol 15, 1, 2014.

9. Ambele, M.A., Dessels, C., Durandt, C., Pepper, M.S. Genome-wide analysis of gene expression during adipogenesis in human adipose-derived stromal cells reveals novel patterns of gene expression during adipocyte differentiation. Stem Cell Res 16, 725, 2016.

10. Miyagi-Shiohira, C., Kobayashi, N., Saitoh, I., et al. Evaluation of serum-free, xeno-free cryopreservation solutions for human adipose-derived mesenchymal stem cells. Cell Med 9, 15, 2017.

11. De Rosa, A., De Francesco, F., Tirino, V., et al. A new method for cryopreserving adipose-derived stem cells: an attractive and suitable large-scale and long-term cell banking technology. Tissue Eng Part C Methods 15, 659, 2009.

12. López, M., Bollag, R.J., Yu, J.C., Isales, C.M., and Eroglu, A. Chemically defined and xeno-free cryopreservation of human adipose-derived stem cells. PLoS One 11, e0152161, 2016.

13. Pittenger, M.F., Mackay, A.M., Beck, S.C., et al. Multilineage potential of adult human mesenchymal stem cells. Science (80-.) 284, 143, 1999.

14. Bruder, S.P., Jaiswal, N., and Haynesworth, S.E. Growth kinetics, self-renewal, and the osteogenic potential of purified human mesenchymal stem cells during extensive subcultivation and following cryopreservation. J Cell Biochem 64, 278, 1997.

15. Bourin, P., Bunnell, B.A., Casteilla, L., et al. Stromal cells from the adipose tissue-derived stromal vascular fraction and culture expanded adipose tissue-derived stromal/stem cells: a joint statement of the International Federation for Adipose Therapeutics and Science (IFATS) and the International So. Cytotherapy 15, 641, 2013.

16. Aldridge, A., Kouroupis, D., Churchman, S., English, A., Ingham, E., and Jones, E. Assay validation for the assessment of adipogenesis of multipotential stromal cells-a direct comparison of four different methods. Cytotherapy 15, 89, 2013.

17. Durandt, C., van Vollenstee, F.A., Dessels, C., et al. Novel flow cytometric approach for the detection of adipocyte sub-populations during adipogenesis. J Lipid Res 57, 729, 2016.

18. van Vollenstee, F.A., Dessels, C., Kallmeyer, K., et al. Isolation and characterization of adipose-derived stromal cells. In: van Pham P., ed. Stem Cell Process. [Internet]. Cham: Springer International Publishing, 2016, pp. 131161.

19. Zuk, P.A., Zhu, M., Mizuno, H., et al. Multilineage Cells from Human Adipose Tissue: implications for Cell-Based Therapies. Tissue Eng 7, 211, 2001.

20. Schallmoser, K., and Strunk, D. Generation of a pool of human platelet lysate and efficient use in cell culture. Methods Mol Biol 946, 349, 2013.

21. Dessels, C., Durandt, C., and Pepper, M.S. Comparison of human platelet lysate alternatives using expired and freshly isolated platelet concentrates for adipose-derived stromal cell expansion. Platelets 30, 356, 2019.

22. Amable, P.R., Teixeira, M.V.T., Carias, R.B.V., Granjeiro, J.M., and Borojevic, R. Identification of appropriate reference genes for human mesenchymal cells during expansion and differentiation. PLoS One 8, e73792, 2013.

23. Fink, T., Lund, P., Pilgaard, L., Rasmussen, J.G., Duroux, M., and Zachar, V. Instability of standard PCR reference genes in adipose-derived stem cells during propagation, differentiation and hypoxic exposure. BMC Mol Biol 9, 1, 2008.

24. Zhang, J., Tang, H., Zhang, Y., Deng, R., Shao, L., Liu, Y., et al. Identification of suitable reference genes for quantitative RT-PCR during 3T3-L1 adipocyte differentiation. Int J Mol Med 33, 1209, 2014.

25. Vandesompele, J., De Preter, K., Pattyn, F., et al. Accurate normalization of real-time quantitative RT-PCR data by geometric averaging of multiple internal control genes. Genome Biol 3, RESEARCH0034, 2002.

26. Perkins, J.R., Dawes, J.M., McMahon, S.B., Bennett DLH, Orengo, C., and Kohl, M. ReadqPCR and NormqPCR: R packages for the reading, quality checking and normalisation of RT-qPCR quantification cycle $(\mathrm{Cq})$ data. BMC Genomics 13, 296, 2012.

27. R Core Team. R: A language and environment for statistical computing. Vienna, Austria: R Foundation for Statistical Computing; 2016; Available from: www.r-project.org/.

28. Doucet, C., Ernou, I., Zhang, Y., et al. Platelet lysates promote mesenchymal stem cell expansion: a safety substitute for animal serum in cell-based therapy applications. J Cell Physiol 205, 228, 2005.

29. Bustin, S., Benes, V., Garson, J., et al. The MIQE guidelines: minimum information for publication of quantitative real-time PCR experiments. Clin Chem 55, 611, 2009.

30. Castanera, R., López-Varas, L., Pisabarro, A.G., and Ramíre, L. Validation of reference genes for transcriptional analyses in Pleurotus ostreatus by using reverse transcription-quantitative PCR. Appl Environ Microbiol 81, 4120, 2015.

31. Chervoneva, I., Li, Y., Schulz, S., et al. Selection of optimal reference genes for normalization in quantitative RTPCR. BMC Bioinformatics 11, 253, 2010.

32. Andersen, C.L., Ledet-Jensen, J., Orntoft, T.F., et al. Normalization of Real-Time quantitative reverse transcription- PCR data: a model-based variance estimation approach to identify genes suited for normalization, applied to bladder and colon cancer data sets. Cancer Res 64, 5245, 2004.

33. De Spiegelaere, W., Dern-Wieloch, J., Weigel, R., et al. Reference gene validation for RT-qPCR, a note on different available software packages. PLoS One 10, 1, 2015.

34. Perez, L.J., Rios, L., Trivedi, P., et al. Validation of optimal reference genes for quantitative real time PCR in muscle and adipose tissue for obesity and diabetes research. Sci Rep 7, 1, 2017.

35. Li, M.-Y., Wang, F., Jiang, Q., Wang, G.-L., Tian, C., and Xiong, A.-S. Validation and comparison of reference genes for qPCR normalization of Celery (Apium graveolens) at different development stages. Front Plant Sci 7, 1, 2016.

36. Vega-Sanchez, R., Arenas-Hernandez, M., Vazquez-Perez, J.A., Moreno-Valencia, Y., and Gomez-Lopez, N. Evaluation of reference genes for expression studies in leukocytes from term human pregnancy. Placenta 36, 240, 2015. 
37. Petriccione, M., Mastrobuoni, F., Zampella, L., and Scortichini, M. Reference gene selection for normalization of RT-qPCR gene expression data from Actinidia deliciosa leaves infected with Pseudomonas syringae pv. Actinidiae Sci Rep 5, 1, 2015.

38. Fox, B.C., Devonshire, A.S., Schutte, M.E., et al. Toxicology in Vitro Validation of reference gene stability for APAP hepatotoxicity studies in different in vitro systems and identification of novel potential toxicity biomarkers. Toxicol Vitr 24, 1962, 2010.

39. Sandercock, D.A., Coe, J.E., Di Giminiani, P., and Edwards, S.A. Determination of stable reference genes for RT-qPCR expression data in mechanistic pain studies on pig dorsal root ganglia and spinal cord. Res Vet Sci 114, 493, 2017.

40. Iyer, G., Wang, A.R., Brennan, S.R., et al. Identification of stable housekeeping genes in response to ionizing radiation in cancer research. Sci Rep 7, 43763, 2017.

41. James, A.W., Levi, B., Nelson, E.R., et al. Deleterious effects of freezing on osteogenic differentiation of human adipose-derived stromal cells in vitro and in vivo. Stem Cells Dev 20, 427, 2011.

42. Yong, K.W., Pingguan-Murphy, B., Xu, F., et al. Phenotypic and functional characterization of long-term cryopreserved human adipose-derived stem cells. Sci Rep 5, 9596, 2015.

43. Dessels, C., Potgieter, M., and Pepper, M.S. Making the switch: alternatives to fetal bovine serum for adipose-derived stromal cell expansion. Front Cell Dev Biol 4, 1, 2016.

44. Koellensperger, E., Bollinger, N., Dexheimer, V., Gramley, F., Germann, G., Leimer, U. Choosing the right type of serum for different applications of human adipose tissuederived stem cells: influence on proliferation and differentiation abilities. Cytotherapy 16, 789, 2014.

45. Trojahn Kølle, S., Oliveri, R.S., Glovinski, P.V., et al. Pooled human lysate versus fetal bovine serumInvestigating the proliferation rate, chromosome stability and angiogenic potential of human adipose tissueederived stem cells intended for clinical use. Cytotherapy 15, 1086, 2013.

46. Riis, S., Nielsen, F., Pennisi, C., Zachar, V., and Fink, T. Comparative analysis of media and supplements on in- ititation and expansion of adipose-derived stem cells. Stem Cells Transl Med 5, 314, 2016.

47. Ben Azouna, N., Jenhani, F., Regaya, Z., et al. Phenotypical and functional characteristics of mesenchymal stem cells from bone marrow: comparison of culture using different media supplemented with human platelet lysate or fetal bovine serum. Stem Cell Res Ther 3, 6, 2012.

48. Blande, I.S., Bassaneze, V., Lavini-Ramos, C., et al. Adipose tissue mesenchymal stem cell expansion in animal serum-free medium supplemented with autologous human platelet lysate. Transfusion 49, 2680, 2009.

49. Portalska, K.J., Groen, N., Krenning, G., et al. The effect of donor variation and senescence on endothelial differentiation of human mesenchymal stromal cells. Tissue Eng Part A 19, 2318, 2013.

50. Ferguson, B.S., Nam, H., Hopkins, R.G., and Morrison, R.F. Impact of reference gene selection for target gene normalization on experimental outcome using real- time qRT-PCR in adipocytes. PLoS One 5, e15208, 2010.

Address correspondence to:

Michael Sean Pepper, MBChB, PhD, MD Institute for Cellular and Molecular Medicine Department of Immunology SAMRC Extramural Unit for Stem Cell Research and Therapy

Faculty of Health Sciences University of Pretoria Prinshof Campus Room 5-64, Level 5, Pathology Building

15 Bophelo Road Pretoria 0084 South Africa

E-mail: michael.pepper@up.ac.za

Received: March 21, 2019

Accepted: May 6, 2019

Online Publication Date: June 6, 2019 